\title{
Espacio universitario español en la Web (2010): estudio descriptivo de instituciones y productos académicos a través del análisis de subdominios y subdirectorios
}

\author{
Enrique Orduña-Malea* \\ *Universidad Politécnica de Valencia (UPV). Valencia (España) \\ Correo-e: enorma@upv.es
}

Recibido: 01-06-2012; 2a versión: 25-07-2012; Aceptado: 26-07-2012

Cómo citar este artículo/ Citation: Orduña-Malea, E. (2013). Espacio universitario español en la Web (2010): estudio descriptivo de instituciones y productos académicos a través del análisis de subdominios y subdirectorios. Revista Española de Documentación Científica, 36(3):e017. doi: http://dx.doi.org/10.3989/redc.2013.3.958

Resumen: Se presenta un análisis descriptivo del sistema universitario español en la Red durante 2010, mediante la identificación, recopilación y análisis de una muestra de entidades y URLs asociados, tanto a nivel de universidad como de unidades universitarias clasificadas en instituciones y productos. Se analiza la cantidad de instituciones con URL válida a efectos de análisis cibermétricos, el tipo de sintaxis de URL subdominio o subdirectorio, así como la detección de prácticas de multidominios y redireccionamientos. Para las unidades con área del conocimiento identificable facultades, escuelas, departamentos, y grupos, centros e institutos de investigación, se realiza igualmente un análisis temático. Los resultados indican que el espacio académico español tiene una estructura compleja, donde abundan las prácticas de redireccionamiento, multidominio, con un predominio de los subdirectorios en las instituciones y subdominios en los productos, y donde las ciencias naturales tienen, en número de entidades y URLs asociados, una presencia mayoritaria.

Palabras clave: Sistema universitario; cibermetría; webometría; instituciones universitarias; productos universitarios; análisis temático; sintaxis de URLs; España.

Spanish university space on the Web (2010): descriptive study of academic institutions and products through the analysis of subdomains and folders

Abstract: A descriptive analysis of the Spanish university system on the Net during 2010 is presented, through the identification, collection and analysis of a sample of entities (and associated URLs), at both the level of universities and university units (classified into institutions and products). An analysis is made of the number of institutions with valid URLs suited for cybermetric analysis purposes and the type of URL syntax (subdomain or subdirectory). Likewise, multi-domain and redirection practices are detected. For units with an identifiable area of knowledge (faculties, schools, departments, and research groups, centers and institutes), a thematic analysis is also carried out. The results indicate that the Spanish academic space has a complex structure, with abundant redirection and multi-domain practices, with a predominance of subdirectories in institutions and subdomains in products, and where the natural sciences have -by number of associated entities and URLS - a major presence.

Keywords: University syste; cybermetrics; webometrics; university institutions; university products; thematic analysis; URL syntax; Spain.

Copyright: ( 2013 CSIC. Este es un artículo de acceso abierto distribuido bajo los términos de la licencia Creative Commons Attribution-Non Commercial (by-nc) Spain 3.0. 


\section{INTRODUCCIÓN}

Una de las áreas de aplicación más profusas de la cibermetría es el análisis de sistemas universitarios a partir del espacio web generado por las universidades que lo componen, debido a que estos espacios constituyen excelentes bancos de prueba tanto para el testeo de ciertos indicadores, especialmente los análisis de enlaces (Thelwall y otros, 2005), como para el análisis indirecto de las propias universidades.

Los primeros trabajos en este sentido se localizan en el Reino Unido, donde Thelwall (2002a) estudia los factores geográficos en el enlazado entre universidades de la zona, descubriendo que la cantidad de enlaces entre pares de universidades decrece con la distancia. Por otra parte, el mismo Thelwall (2002b) analiza la relación entre la cantidad de enlaces y la producción científica, encontrando una correlación significativa, que no se da en otros sistemas universitarios, como el español (Thelwall y Aguillo, 2003). Igualmente destaca el análisis del espacio académico escandinavo (Ortega y Aguillo, 2008).

Tomando Europa en su conjunto, destacan los estudios de Boudorides y otros (1999), Polanco y otros (2001), Thelwall y otros (2002) y Ortega y otros (2008), quienes visualizan las relaciones y redes entre universidades a partir de sus sedes web. Igualmente, se detectan algunos otros trabajos centrados en la identificación de ciertos patrones culturales a través de la información contenida en las sedes webs académicas, como Heimeriks y otros (2003), quienes mapean 220 universidades europeas detectando patrones culturales y lingüísticos en sus relaciones, y Thelwall y otros (2003), quienes identifican disparidades en los tamaños web entre el Oeste y Este de Europa.

Fuera de Europa, se pueden destacar los numerosos análisis de universidades asiáticas y orientales, como Israel (Bar-Ilan, 2003), China (Qiu y otros, 2004) e Irán (Kousha y Horri, 2004), así como de áreas más amplias como todo el oriente medio (Noruzi, 2006) e incluso de las relaciones de Asia con Europa (Park y Thelwall, 2006).

En América destacan fundamentalmente los trabajos realizados en los países suramericanos, donde merece atención especial el análisis del espacio académico chileno realizado por Baeza-Yates y Graells ${ }^{1}$ y los trabajos de González Martín y Aguillo (1999), Aguillo (2005) y Ortega y Aguillo (2009a).

Norteamérica y Canadá, pese a la importancia de sus sistemas universitarios, han sido muy escasamente estudiados, destacando el trabajo de Ortega y Aguillo (2009b). Respecto a Australia, destaca el trabajo realizado por Smith y Thelwall (2002), mientras que el continente africano ha sido estudiado solo parcialmente (Nwagwu y Agarin, 2008; Adecannby, 2011).
A nivel mundial, destaca especialmente el trabajo realizado por el Cybermetrics Lab en la confección del Ranking Web de Universidades del mundo (Aguillo y otros, 2006).

Respecto a España, éste es un sistema ampliamente analizado, estudiado por Pinto-Molina y otros (2004), Ortega y Aguillo (2007), Orduña-Malea y otros (2009) y Orduña-Malea y otros (2010) y, de manera tangencial, por Martínez-Torres y otros (2011).

En todos estos trabajos la unidad de análisis es la universidad, y en pocos casos se llega a un nivel inferior, donde los patrones de enlazado (u otros indicadores) a veces no siguen las mismas pautas que a nivel universitario. Los departamentos son las unidades más estudiadas, donde sobresalen los trabajos de Thomas y Willet (2000), Thelwall (2002c, 2003), Tang y Thelwall (2003, 2004), y muy especialmente Li (2005).

En cuanto al caso español, merece especialmente su atención la tesis doctoral de Ortega (2007), quien identifica y describe el rendimiento, además de universidades, de grupos y departamentos universitarios.

Sin embargo, se detecta una carencia de estudios que aborden una casuística más amplia de unidades universitarias web internas, lo que permitiría conocer mejor la estructura del espacio académico universitario web.

\section{OBJETIVOS}

El principal objetivo de este trabajo es realizar un análisis descriptivo del sistema universitario español en la Red durante 2010, mediante la identificación, recopilación y análisis de una muestra de entidades y URLs asociados, estructurados en función de las diversas misiones universitarias.

\section{METODOLOGÍA}

\subsection{Método de análisis descriptivo}

En este apartado se describe el proceso de recopilación de URLs, su estructuración y análisis cuantitativo descriptivo.

\subsubsection{Obtención y estructuración de la muestra}

\section{a) Nivel institucional de contorno}

El modelo de análisis se aplica al Sistema Universitario Español (SUE), formado en 2010 por 76 universidades, tanto públicas como privadas. Tanto el listado de universidades como de sus URLS se obtuvieron del Ministerio de Educación ${ }^{2}$ y de la Conferencia de Rectores de las Universidades Españolas ${ }^{3}$.

Una vez recogidas y clasificadas las distintas universidades, se recopiló el URL correspondiente a cada una de ellas (anexo I). Además de los URLs 
indicados en las fuentes oficiales consultadas, se comprobó la existencia de dominios "alias" y dominios "alternativos" en diversas universidades. Para realizar el estudio de forma exhaustiva se determinó recopilar todos estos URLs, aunque redireccionaran a otro URL, o no tuvieran apenas información alojada.

Los dominios "alias" son URLs que comparten el mismo dominio de segundo nivel, pero tienen distintos dominios de primer nivel. Para localizar estos URLs se comprobó manualmente en cada universidad la existencia de los siguientes dominios: .CAT, .COM, .EDU., ES, .NET, .ORG.

De forma alternativa, se localizaron dominios activos para ciertas universidades que no constituían "alias" sino dominios web completamente distintos (en adelante dominios alternativos). Por ejemplo, en el caso de la Universidad Camilo José Cela, se localizaron por una parte, los dominios alias "ucjc. es" y "ucjc.edu", y por otra parte, el dominio "universidadcamilojosecela.es", donde el nombre de dominio de segundo nivel es diferente.

Para localizar estos dominios alternativos se realizaron búsquedas de enlaces a través de Yahoo! Site Explorer, además de consultar la propia información institucional de la universidad en su sede web.

\section{b) Nivel institucional de unidad}

En primer lugar se procedió a estructurar internamente la universidad en función de sus activi-

Tabla I. Entidades y productos universitarios dades y objetivos. En este caso, las actividades consideradas fueron las siguientes: docencia, investigación, transferencia, servicios y administración. Estas actividades explicitan el carácter multidimensional de la universidad.

Aparte de las 3 misiones clásicas, se añadieron 2 actividades complementarias (servicios y administración), que si bien no son objetivos primarios de la institución (misiones), sí son funciones que permiten su correcto funcionamiento, además de generar una amplia cantidad de documentación en Red.

Una vez consideradas las actividades fundamentales, se identificaron y clasificaron los distintos tipos de entidades existentes dentro de una universidad, que se dividieron en dos clases: instituciones (departamentos, facultades, grupos de investigación, etc.) y productos (repositorios institucionales, campus virtuales, plataformas multimedia y de blogs, etc.).

En el caso de las bibliotecas y los archivos, se separó la sede web de estas instituciones del catálogo o fondo documental, en el caso de que vía web estuvieran diferenciados. De esta forma, una biblioteca se considera como entidad institución (adscrita a la actividad de "servicios"), mientras que el OPAC, si tiene URL identificable e independiente, se considera como entidad producto, también adscrita a "servicios".

La tabla I muestra todos los tipos de entidades recogidos finalmente en este estudio, asignados a cada una de las 5 actividades principales.

\begin{tabular}{|c|c|}
\hline ACTIVIDAD & TIPO DE ENTIDAD \\
\hline \multirow{3}{*}{ Docencia } & Aulas virtuales \\
\hline & Departamentos \\
\hline & Plataformas OpenCourseWare (OCW) \\
\hline \multirow{4}{*}{ Investigación } & Centros de investigación \\
\hline & Grupos de investigación \\
\hline & Institutos de investigación \\
\hline & Repositorios institucionales \\
\hline \multirow{2}{*}{ Transferencia } & Centros de formación permanente \\
\hline & Oficinas de Transferencia de Resultados de investigación (OTRI) \\
\hline \multirow{8}{*}{ Servicios } & Archivos \\
\hline & Asociaciones de antiguos alumnos y amigos \\
\hline & Bibliotecas \\
\hline & Centros de Documentación Europea (CDE) \\
\hline & Institutos de Ciencias de la Educación (ICE) \\
\hline & Plataformas multimedia \\
\hline & Plataformas de blogs \\
\hline & Catálogos y fondos documentales \\
\hline \multirow{6}{*}{ Administración } & Centros culturales y de estudios \\
\hline & Escuelas de negocios \\
\hline & Escuelas Universitarias \\
\hline & Facultades \\
\hline & Fundación universidad \\
\hline & Vicerrectorados \\
\hline
\end{tabular}


En total se consideraron 23 tipos de entidades (17 tipos de instituciones y 6 tipos de productos). El criterio para su elección fue, en el caso de las instituciones, que la creación de éstas estuviera regulada y legislada. En el caso de los productos, se recogieron aquellos que tenían una presencia en al menos 10 universidades diferentes, y que fueran susceptibles de generar grandes cantidades de documentación.

Obviamente, existen muchas más instituciones y productos universitarios (cátedras universidadempresa, campus de excelencia, etc.), pues la universidad es una institución muy diversa y compleja, pero los 23 tipos de entidades identificados reflejan un porcentaje muy elevado de la producción universitaria, además de representar la actividad esencial universitaria.
Tras la identificación y selección de los tipos de entidades a estudiar, se procedió a recopilar todas las instituciones y productos existentes en cada una de las 76 universidades del SUE.

Para localizar las diferentes entidades internas de la universidad se procedió en primer lugar a navegar por las diferentes sedes web universitarias (ejemplo, figura 1).

La información completa de entidades no siempre se encontraba centralizada en el tipo de página mostrada en la figura 1 , por lo que fue necesario un proceso manual de navegación dentro de cada sede, la comprobación de la existencia de cada entidad localizada así como su correcta adscripción a cada tipo de entidad.

Se utilizaron igualmente las siguientes fuentes de información (tabla II) ${ }^{4}$ :

Figura 1. Ejemplo de localización de entidades en la UA

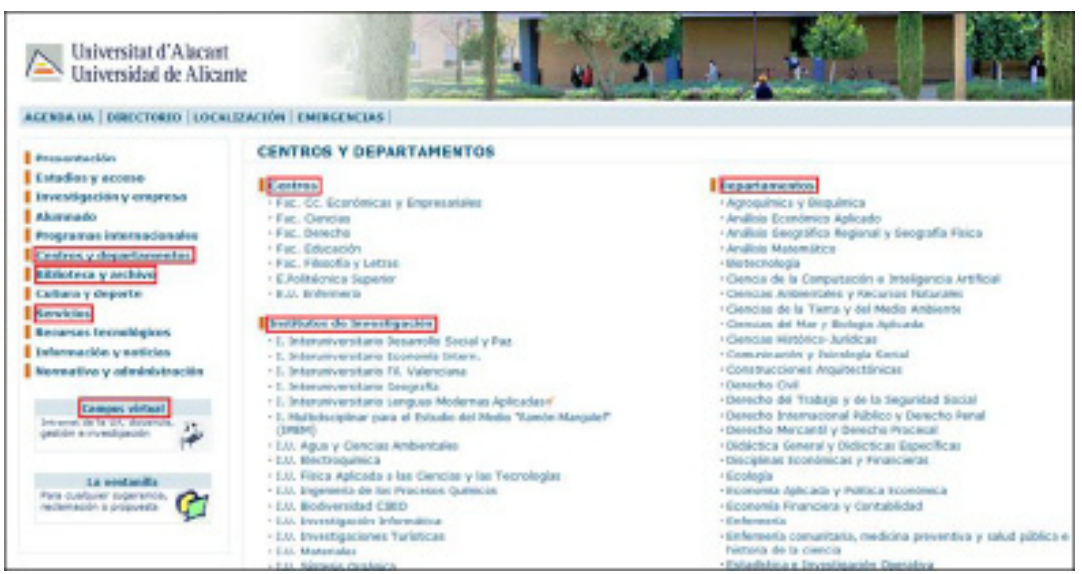

Fuente: http://www.ua.es

Tabla II. Fuentes de información de universidades

\begin{tabular}{|c|c|}
\hline FUENTE & URL \\
\hline Archivos universitarios españoles & uclm.es/archivo/mapas/mapaes.asp \\
\hline Asociación Española de fundaciones & fundaciones.org \\
\hline Busca Repositorios & accesoabierto.net/repositorios \\
\hline Comisión Europea & ec.europa.eu/spain/redes/cde/index_es.htm \\
\hline Federación de asociaciones de antiguos alumnos & faaaa.universia.es \\
\hline Madri+d - OTRIS & madrimasd.org/informacionidi/biblioteca/enlacesIDI/otrisID.asp \\
\hline $\begin{array}{l}\text { Ministerio de Cultura - Estructuras de investigación } \\
\text { universitarias }\end{array}$ & $\begin{array}{l}\text { educacion.es/educacion/universidades/investigacion/agentes- } \\
\text { investigacion/estructura-investigacion.html }\end{array}$ \\
\hline $\begin{array}{l}\text { Ministerio de Educación - Centro de Investigación y } \\
\text { Documentación Educativa (CIDE) }\end{array}$ & educacion.es/cide/jsp/plantilla.jsp?id=inv09d \\
\hline Open CourseWare Consortium & ocwconsortium.org \\
\hline Red de Bibliotecas Universitarias Españolas (REBIUN) & rebiun.org \\
\hline Red OTRI & redotriuniversidades.net \\
\hline Universia - OCW & ocw.universia.net/es \\
\hline
\end{tabular}


Adicionalmente, y para completar la búsqueda, se utilizó el comando especial "feature:index" en el buscador Yahoo! Search, para localizar sedes web no reseñadas en las fuentes anteriores, especialmente en el caso de los grupos de investigación.

Para cada una de las instituciones y productos recopilados, se procedió posteriormente a recoger el URL que las representaba.

La unidad de análisis se fijó en el nivel de "sede web", con lo que solo se aceptaron los URLs (y por tanto, las entidades y productos correspondientes) que cumplieran con los siguientes criterios:

- Cada entidad debía corresponder a una sede web desde el punto de vista documental, es decir, ser un subdirectorio o subdominio dentro del dominio académico general web. Esto elimina a todas las entidades con dominio externo al académico y a todas aquellas que constan únicamente de una página web (un solo fichero HTML o XML), en lugar de una sede propiamente dicha.

- El URL que identifica a la sede web no debía ser dinámico, pues en ese caso la jerarquía documental se pierde y a nivel automático es imposible determinar la pertenencia de una página a una determinada unidad documental.

- La sede web debía corresponder o asociarse a uno de los tipos de entidad universitaria seleccionados de forma clara e inequívoca.

Para todas las entidades que cumplían estos requisitos, se recogieron los URLs correspondientes incluyendo -al igual que en el nivel de contorno- los diferentes alias y dominios alternativos. En cada caso, se tomó el subdirectorio o subdominio válido que estuviera en el nivel jerárquico más superior, y que identificara a la institución o producto en cuestión. Todas las entidades cuyos URLs no cumplieron estos requisitos fueron eliminadas del estudio.

En algunos casos se dio la circunstancia de que al tomar el subdirectorio o subdominio de nivel más alto (siempre que fuera válido), este URL no permitía el acceso directo al recurso web a través del navegador. Por ejemplo, al tomar un subdirectorio es posible que éste no redirija al fichero "index" y aparezca en pantalla o bien el índice del directorio del hosting web o la pantalla en blanco, e incluso que, por cuestiones de seguridad, no proporcione acceso y redirija a otro URL. Por ejemplo, el URL del Grupo interdisciplinar de física de fluidos complejos ${ }^{5}$, de la UAL, cuya sintaxis es de tipo directorio, no redirige a la página de inicio del grupo, aunque es el URL que identifica a nivel jerárquico más elevado la entidad.

En el caso de las redirecciones, se tomaron las siguientes medidas:

- Si un URL (A) válido, redirige a otro no válido (B), se tomó el primero, pues es susceptible de recibir enlaces externos, y se desechó el segundo.

- Si un URL (A) válido, redirige a otro válido (B), se tomaron los dos.

- Si un URL localizado no funcionaba o no dirigía a ningún recurso (enlace roto), pero su sintaxis era válida, se tomó en cuenta pues era igualmente susceptible de recibir enlaces externos.

Tras aplicar los procesos descritos anteriormente, se obtuvieron finalmente 2 muestras para el análisis, la correspondiente a entidades (subdividida en instituciones y productos) y la de sus URLS (igualmente subdividida en instituciones y productos). Estas 4 muestras se volcaron en diversas hojas de cálculo, donde se estructuraron, describieron y normalizaron.

El proceso de búsqueda, recolección, estructuración y normalización, tanto de las entidades como de los URLs, se realizó en una primera toma entre enero y marzo de 2010. Posteriormente se realizaron 3 actualizaciones de carácter trimestral (junio, septiembre y diciembre de 2010) para recoger nuevas muestras así como para corregir posibles errores ${ }^{6}$.

En el caso de la muestra de entidades, cada entidad identificada con URL válido se clasificó en función de su clase (institución o producto), tipo (departamento, grupo, escuela, etc.) y tipo de universidad (pública o privada).

En el caso de los centros de investigación, institutos de investigación, grupos de investigación, departamentos, facultades y escuelas, se procedió adicionalmente a clasificarlos temáticamente en función de sus áreas de especialización asociadas. A partir de la clasificación temática de la Agencia Nacional de Evaluación y Prospectiva (ANEP) ${ }^{7}$, se optó por agregar los campos para obtener una clasificación genérica de 5 grandes bloques, lo suficientemente amplia y general como para permitir la observación de las posibles diferencias en la presencia web según las distintas áreas de actividad humana, sin ninguna mayor pretensión clasificatoria. La nomenclatura de la UNESCO para áreas temáticas, utilizada por ejemplo en la tesis doctoral de Ortega (2007), se desestimó, pues los grupos de investigación, departamentos y otras unidades se adscriben más fácilmente a grandes áreas generales que a categorías más específicas (la Nomenclatura de la UNESCO establece 28 categorías).

Las áreas que se establecieron fueron las siguientes:

- Arte y humanidades (AHU): Historia del Arte, Geografía, Historia, Filologías...

- Ciencias formales (CFO): Filosofía, Lógica, Matemáticas...

- Ciencias naturales (CNA): Química, Física, Biología, Medicina, Ciencias de la salud... 
- Ciencias sociales (CSO): Derecho, Economía, Política, Sociología, Documentación...

- Ingeniería (ING): Industriales, Informática, Telecomunicaciones, Electrónica, Aeronáutica, Arquitectura, Acústica, Óptica...

Adicionalmente, cada URL se clasificó, en función de su sintaxis, en subdirectorio o subdominio.

Pese a que es de interés comparar los valores acumulados a nivel interno con los de contorno para conocer qué porcentaje representan los primeros del total, existe un solapamiento importante entre los URLs internos que invalida este proceso.

Muchos grupos de investigación poseen sus URLS dentro de los subdominios de las facultades y departamentos( u otras combinaciones). Por ejemplo, el Grupo de métodos numéricos en ingeniería, de la UDC, posee su URL dentro del subdominio de la Escuela Técnica Superior de Ingenieros de Caminos, Canales y Puertos $^{8}$. Esta mala práctica en la gestión de DNSs académicos provoca la necesidad de un proceso de limpieza de datos que afecta al análisis de unidades, fundamentalmente a las medidas de tamaño (las medidas de enlazado son filtradas automáticamente por Yahoo Site Explorer).

Por esta razón, los datos comparativos de tamaño interno/contorno no se presentan formalmente en el trabajo.

\section{RESULTADOS}

Tras la aplicación del proceso de identificación y recopilación descrito en el apartado metodológico, se obtienen dos muestras de datos (de entidades y de URLs), que se analizan a continuación.

El número total de entidades consideradas (divididas en instituciones y productos) es de 7.464, que generan un total de 13.941 direcciones web diferentes. Los datos finales resumidos de entidades y URLs se detallan en la tabla III.

A continuación se detallan los resultados a nivel topológico alcanzados por estas dos muestras de datos en los 2 niveles de análisis.

\subsection{Nivel institucional de contorno}

A fecha de enero de 2011, el sistema universitario español se compone de un total de 76 universidades, de las que $49(64,47 \%)$ son de carácter público y las 27 restantes $(35,53 \%)$ de titularidad privada.

El número total de URLs válidos localizados (es decir, accesibles e institucionales), asociadas a alguna de las 76 universidades, asciende a $141^{9}$, de las que 81 corresponden a universidades públicas y 60 a privadas. Estos datos indican un uso moderado-alto de multidominios.

De las 76 universidades españolas solo 47 poseen un único dominio web oficial ${ }^{10}$, mientras que 29 (el 38,16\%) poseen al menos dos dominios válidos. Estas 29 universidades (14 públicas y 15 privadas) generan los 65 URLs de "exceso" (141 URLs para 76 universidades).

El entorno universitario catalán es el más complejo pues todas sus universidades poseen multidominios. La UAO, UAB, UDL, UVIC disponen de 2 dominios válidos cada una; la UB, UDG, UPC, UPF, URL, URV tienen 3 dominios; finalmente, la UIC y la UOC tienen hasta 4 dominios cada una.

Otros casos especiales de utilización de multidominios son los que presentan la UC3M y la UNAV, con 5 dominios cada una, la UPCO con 6 dominios, y finalmente la UO, para la que se han detectado hasta 8 dominios (tanto alias como alternativos), constituyendo el espacio web más complejo en el entorno universitario español en el período de estudio.

Respecto al uso de redirecciones, de los 65 URLs de exceso, 33 redirigen automáticamente al URL oficial mientras que las 32 restantes coexisten con los oficiales sin ningún tipo de redirección. Los 33 URLs con redirección pertenecen a 15 universidades ( 7 públicas y 8 privadas), que suponen un $19,74 \%$ del total del espacio universitario web.

Finalmente, 4 URLs (unileon.org; uvic.es; urjc. net; uan.es), válidos en enero de 2010, dejan de ser accesibles a lo largo del año, no proporcionan-

Tabla III. Resumen de instituciones y URLs recopiladas

\begin{tabular}{|c|c|c|c|c|c|c|}
\hline \multirow{2}{*}{ NIVEL } & \multicolumn{3}{|c|}{ ENTIDADES } & \multicolumn{3}{|c|}{ URLS } \\
\hline & INSTITUCIÓN & PRODUCTO & $\mathbf{N}$ & INSTITUCIÓN & PRODUCTO & $\mathbf{N}$ \\
\hline CONTORNO & $x$ & $x$ & 76 & $x$ & $x$ & 141 \\
\hline UNIDAD & 7.098 & 293 & 7.391 & 13.417 & 383 & 13.800 \\
\hline TOTAL & $\mathbf{x}$ & $\mathbf{x}$ & 7.464 & $\mathbf{x}$ & $\mathbf{x}$ & 13.941 \\
\hline
\end{tabular}


do acceso en diciembre de 2010 a la universidad correspondiente.

\subsection{Nivel institucional de unidad}

El análisis descriptivo a nivel de unidad se divide en dos bloques principales correspondientes a los dos tipos de entidades estudiadas: instituciones y productos. A su vez, cada uno de estos bloques se subdivide en una muestra de entidad y otra de URLS.

\subsubsection{Muestra de instituciones}

En este apartado se analizan cuantitativamente todas las instituciones recopiladas en función de cada uno de los 17 tipos de instituciones, así como los URLs que estas instituciones generan.

\section{a) Análisis general deentidades (instituciones)}

Se han obtenido un total de 7.098 ítems, cuya distribución por "tipo de institución" puede observarse en la tabla IV.

Los grupos de investigación y los departamentos son las entidades con más ítems (2.867 y 2.179 respectivamente), lo que es un resultado lógico puesto que cada universidad dispone por regla general, de una gran cantidad de este tipo de instituciones en contraposición a otras instituciones como los archivos, fundaciones, centros de documentación europea o asociaciones de antiguos alumnos que, incluso por definición legal en algunos casos, solo disponen de un ítem por universidad.
En el caso de las escuelas de negocios, éstas se implantan tradicionalmente fuera del entorno universitario (centros de formación privados externos y ajenos a la universidad), por lo que su presencia en las universidades -sobre todo en España- es esporádica, como ejemplifican las 12 instituciones localizadas.

La tabla $\mathrm{V}$ ordena las universidades en función del número total de instituciones válidas recopiladas. De forma complementaria se muestra el número de dominios -a nivel de contorno-identificados para cada universidad (con el fin de contextualizar los datos por tamaño) y el tipo de universidad de que se trata (pública o privada).

Solamente 3 universidades (UDIMA, UNIR y VIU) quedan sin ítems identificados a nivel interno (y por tanto sin URLs). Estas universidades ejemplifican la baja posición general lograda por las universidades privadas (la universidad privada con más instituciones es la UNAV, en la posición 26). Por otra parte, se destaca la proximidad de las 3 politécnicas más importantes (UPV, UPC y UPM), quedando la UPCT algo más relegada.

Otros resultados de interés son las altas posiciones logradas por la UCA, UNEX y, sobre todo, por la UO, en primera posición de forma destacada, por delante de la UCM, que ocupa el segundo lugar, aunque solo utiliza 1 dominio, por los 8 identificados para la UO.

Tabla IV. Distribución de instituciones por tipo de entidad

\begin{tabular}{|c|c|c|c|}
\hline TIPO DE INSTITUCIONES & $\mathbf{N}$ & \% (institución) & $\%$ (total) \\
\hline Grupos de investigación (GIN) & 2.867 & 40,39 & 38,79 \\
\hline Departamentos universitarios (DEP) & 2.179 & 30,70 & 29,48 \\
\hline Facultades universitarias (FAC) & 486 & 6,85 & 6,58 \\
\hline Institutos de investigación (INS) & 337 & 4,75 & 4,56 \\
\hline Escuelas universitarias (ESC) & 307 & 4,33 & 4,15 \\
\hline Vicerrectorados (VIC) & 265 & 3,73 & 3,59 \\
\hline Centros de investigación (CIN) & 146 & 2,06 & 1,98 \\
\hline Bibliotecas universitarias (BIB) & 116 & 1,63 & 1,57 \\
\hline Asociaciones de antiguos alumnos y amigos (AAA) & 76 & 1,07 & 1,03 \\
\hline Centros de estudios (CES) & 71 & 1,00 & 0,96 \\
\hline Oficinas de transferencia de resultados (OTRI) & 52 & 0,73 & 0,70 \\
\hline Fundaciones universitarias (FUN) & 44 & 0,62 & 0,60 \\
\hline Centros de formación posgrado (CFP) & 41 & 0,58 & 0,55 \\
\hline Archivos universitarios (ARC) & 37 & 0,52 & 0,50 \\
\hline Institutos de ciencias de la educación (ICE) & 34 & 0,48 & 0,46 \\
\hline Centros de documentación europea (CDE) & 28 & 0,39 & 0,38 \\
\hline Escuelas de negocios (BS) & 12 & 0,17 & 0,16 \\
\hline TOTAL & 7.098 & 100 & 96,04 \\
\hline
\end{tabular}


Tabla V. Universidades en función del número de instituciones con sede web

\begin{tabular}{|c|c|c|c|c|c|}
\hline $\mathbf{R}$ & UNIVERSIDAD & $\mathbf{N}$ & $\%$ & DOM & TIPO \\
\hline 1 & Universidad de Oviedo (UO) & 407 & 5,73 & 8 & PUB \\
\hline 2 & Universidad Complutense de Madrid (UCM) & 348 & 4,90 & 1 & PUB \\
\hline 3 & Universidad de Granada (UGR) & 263 & 3,71 & 1 & PUB \\
\hline 4 & Universidad del País Vasco (EHU) & 258 & 3,63 & 1 & PUB \\
\hline 5 & Universidad de Cádiz (UCA) & 247 & 3,48 & 1 & PUB \\
\hline 6 & Universidad de Extremadura (UNEX) & 247 & 3,48 & 1 & PUB \\
\hline 7 & Universidad de Santiago de Compostela (USC) & 213 & 3,00 & 1 & PUB \\
\hline 8 & Universidad de Barcelona (UB) & 210 & 2,96 & 3 & PUB \\
\hline 9 & Universidad de Valencia (UV) & 206 & 2,90 & 1 & PUB \\
\hline 10 & Universidad de Sevilla (US) & 197 & 2,78 & 1 & PUB \\
\hline 11 & Universidad Politécnica de Valencia (UPV) & 186 & 2,62 & 1 & PUB \\
\hline 12 & Universidad Autónoma de Madrid (UAM) & 179 & 2,52 & 1 & PUB \\
\hline 13 & Universidad de Zaragoza (UZ) & 178 & 2,51 & 1 & PUB \\
\hline 14 & Universidad Politécnica de Cataluña (UPC) & 176 & 2,48 & 3 & PUB \\
\hline 15 & Universidad de Murcia (UM) & 170 & 2,40 & 1 & PUB \\
\hline 16 & Universidad Politécnica de Madrid (UPM) & 169 & 2,38 & 1 & PUB \\
\hline 17 & Universidad Autónoma de Barcelona (UAB) & 149 & 2,10 & 2 & PUB \\
\hline 18 & Universidad de Alicante (UA) & 146 & 2,06 & 1 & PUB \\
\hline 19 & Universidad de Castilla-La Mancha (UCLM) & 142 & 2,00 & 5 & PUB \\
\hline 20 & Universidad de Málaga (UMA) & 140 & 1,97 & 1 & PUB \\
\hline 21 & Universidad de La Coruña (UDC) & 133 & 1,87 & 1 & PUB \\
\hline 22 & Universidad de Salamanca (USAL) & 130 & 1,83 & 1 & PUB \\
\hline 23 & Universidad Carlos III de Madrid (UC3M) & 128 & 1,80 & 5 & PUB \\
\hline 24 & Universidad de Valladolid (UVA) & 128 & 1,80 & 1 & PUB \\
\hline 25 & Universidad de Vigo (UVI) & 127 & 1,79 & 1 & PUB \\
\hline 26 & Universidad de Navarra (UNAV) & 121 & 1,70 & 5 & PRI \\
\hline 27 & Universidad de Córdoba (UCO) & 116 & 1,63 & 1 & PUB \\
\hline 28 & Universidad Jaume I (UJI) & 113 & 1,59 & 1 & PUB \\
\hline 29 & Universidad de Gerona (UDG) & 109 & 1,54 & 3 & PUB \\
\hline 30 & Universidad de Cantabria (UC) & 102 & 1,44 & 1 & PUB \\
\hline 31 & Universidad de Las Palmas de Gran Canaria (ULPGC) & 98 & 1,38 & 1 & PUB \\
\hline 32 & Universidad de Alcalá (UAL) & 97 & 1,37 & 1 & PUB \\
\hline 33 & Universidad Rovira $i$ Virgili (URV) & 94 & 1,32 & 3 & PUB \\
\hline 34 & Universidad de Jaén (UJA) & 87 & 1,23 & 1 & PUB \\
\hline 35 & Universidad de Burgos (UBU) & 84 & 1,18 & 1 & PUB \\
\hline 36 & Universidad de La Laguna (ULL) & 80 & 1,13 & 1 & PUB \\
\hline 37 & Universidad Pompeu Fabra (UPF) & 80 & 1,13 & 3 & PUB \\
\hline 38 & Universidad de Huelva (UHU) & 72 & 1,01 & 1 & PUB \\
\hline 39 & Universidad Nacional de Educación a Distancia (UNED) & 72 & 1,01 & 1 & PUB \\
\hline 40 & Universidad de Almería (UAL) & 70 & 0,99 & 1 & PUB \\
\hline 41 & Universidad de las Islas Baleares (UIB) & 66 & 0,93 & 2 & PUB \\
\hline 42 & Universidad Rey Juan Carlos (URJC) & 66 & 0,93 & 2 & PUB \\
\hline 43 & Universidad Politécnica de Cartagena (UPCA) & 63 & 0,89 & 1 & PUB \\
\hline 44 & Universidad Internacional de Cataluña (UIC) & 61 & 0,86 & 4 & PRI \\
\hline 45 & Universidad de Lérida (UDL) & 57 & 0,80 & 2 & PUB \\
\hline 46 & Universidad de Mondragón (MU) & 49 & 0,69 & 1 & PRI \\
\hline 47 & Universidad de La Rioja (UR) & 45 & 0,63 & 3 & PUB \\
\hline 48 & Universidad Pública de Navarra (UPNA) & 45 & 0,63 & 1 & PUB \\
\hline 49 & Universidad Pablo de Olavide (UPO) & 42 & 0,59 & 1 & PUB \\
\hline 50 & Universidad de Deusto (UDE) & 38 & 0,54 & 1 & PRI \\
\hline 51 & Universidad Miguel Hernández de Elche (UMH) & 38 & 0,54 & 1 & PUB \\
\hline 52 & Universidad Pontificia de Salamanca (UPSA) & 32 & 0,45 & 1 & PRI \\
\hline 53 & Universidad de León (UL) & 31 & 0,44 & 2 & PUB \\
\hline 54 & Universidad Oberta de Catalunya (UOC) & 25 & 0,35 & 4 & PRI \\
\hline 55 & Universidad Ramon Llull (URL) & 24 & 0,34 & 3 & PRI \\
\hline 56 & Universidad Católica San Antonio de Murcia (UCAM) & 16 & 0,23 & 1 & PRI \\
\hline 57 & Universidad Cardenal Herrera CEU (UCH) & 15 & 0,21 & 3 & PRI \\
\hline 58 & Universidad Europea de Madrid (UEM) & 14 & 0,20 & 1 & PRI \\
\hline 59 & Universidad San Pablo CEU (USP) & 13 & 0,18 & 3 & PRI \\
\hline 60 & IE Universidad (IE) & 11 & 0,15 & 1 & PRI \\
\hline 61 & Universidad de Vic (UVIC) & 11 & 0,15 & 2 & PRI \\
\hline 62 & Universidad Antonio de Nebrija (UAN) & 10 & 0,14 & 2 & PRI \\
\hline 63 & Universidad Europea Miguel de Cervantes (UEMC) & 10 & 0,14 & 3 & PRI \\
\hline 64 & Universidad San Jorge (USJ) & 10 & 0,14 & 4 & PRI \\
\hline 65 & Universidad Alfonso X El Sabio (UAX) & 8 & 0,11 & 1 & PRI \\
\hline 66 & Universidad Pontificia de Comillas (UPCO) & 8 & 0,11 & 6 & PRI \\
\hline 67 & Universidad Católica de Valencia (UCV) & 6 & 0,08 & 1 & PRI \\
\hline 68 & Universidad Abat Oliba CEU (UAO) & 4 & 0,06 & 2 & PRI \\
\hline 69 & Universidad Católica de Ávila (UCAV) & 2 & 0,03 & 1 & PRI \\
\hline 70 & Universidad Francisco de Vitoria (UFV) & 2 & 0,03 & 1 & PRI \\
\hline 71 & Universidad Internacional de Andalucía (UIA) & 2 & 0,03 & 1 & PUB \\
\hline 72 & Universidad Camilo José Cela (UCJC) & 1 & 0,01 & 3 & PRI \\
\hline 73 & Universidad Internacional Menéndez Pelayo (UIMP) & 1 & 0,01 & 3 & PRI \\
\hline 74 & Universidad a Distancia de Madrid (UDIMA) & $\mathbf{0}$ & 0,00 & 1 & PRI \\
\hline 75 & Universidad Internacional de La Rioja (UNIR) & $\mathbf{0}$ & 0,00 & 1 & PRI \\
\hline 76 & Universidad Internacional de Valencia (VIU) & $\mathbf{0}$ & 0,00 & 2 & PRI \\
\hline & TOTAL & 7.098 & 100 & 141 & \\
\hline
\end{tabular}


La tabla VI incluye, por su parte, el análisis temático realizado en las instituciones que tienen asignadas áreas temáticas de investigación y/o docencia, que suponen 6.321 instituciones (cerca del $90 \%$ del total). Se incide en el hecho de que este análisis se hace solo en función de las entidades con presencia web y URL válido, por lo que supone solo un reducido reflejo del sistema universitario a través de la información difundida en la Web.

Los datos muestran una mayor presencia de las ciencias naturales, sociales e ingeniería en detrimento de las artes y humanidades y las ciencias formales, infrarepresentadas sobre todo en lo que respecta a escuelas y facultades. No obstante, cada tipo de entidad presenta distribuciones temáticas diferentes; las ciencias sociales predominan, en proporción, en las facultades (el $49,18 \%$ de las facultades se adscribe a este campo) e institutos de investigación $(38,99 \%)$, las ciencias naturales en los grupos de investigación $(34,84 \%)$ y departamentos $(30,34 \%)$, y las ingenierías en las escuelas $(63,52 \%)$ y centros de investigación $(40,41 \%)$. La ingeniería es además la área menos representada en facultades (es la única área con menor representatividad en facultades que en escuelas).

\section{b) Análisis general de dominios web (insti-} tuciones)

El número total de URLs asociados a las entidades recuperadas asciende a 13.417 (tabla VII). El ranking de tipos de instituciones por número de URLs es prácticamente idéntico al ranking de tipos de instituciones por número de entidades (tabla IV). Solamente los centros de estudios (que superan a las asociaciones de antiguos alumnos) y los institutos de ciencias de la educación (que superan a los archivos, centros de formación y fundaciones) presentan cambios en sus posiciones.

La distribución de URLs es muy desigual. Entre los grupos y los departamentos se obtiene más del $70 \%$ del total de la muestra de universidades, quedando el resto de instituciones muy lejos en representatividad.

Por otra parte, la tabla VII también ofrece información acerca de la naturaleza del URL (subdominio o subdirectorio). En este caso, se observa un claro predominio de los subdirectorios $(83,04 \%$ del total de URLs de instituciones) frente a los subdominios. Solo en el caso de las fundaciones y las escuelas de negocio el balance se compensa.

La tabla VIII ofrece, por otra parte, el ranking de universidades en función del número de URLS totales internos institucionales.

Se observan algunas diferencias respecto al ranking de entidades (tabla V). La UO sigue siendo la institución que ocupa el primer lugar pero se produce un aumento considerable de 2 universidades privadas (UIC y UNAV), cuyas cantidades de URLS

Tabla VI. Áreas temáticas por tipo de institución

\begin{tabular}{lcccccccc}
\hline \multirow{2}{*}{ ÁREA } & \multicolumn{8}{c}{ ENTIDADES (INSTITUCIONES) } \\
\cline { 2 - 11 } & CIN & DEP & ESC & FAC & GIN & INS & N & \% \\
\hline Ciencias naturales & 34 & 661 & 54 & 135 & 999 & 98 & $\mathbf{1 . 9 8 1}$ & $\mathbf{3 1 , 3 4}$ \\
\hline Ciencias sociales & 38 & 654 & 56 & 239 & 670 & 131 & $\mathbf{1 . 7 8 8}$ & $\mathbf{2 8 , 2 9}$ \\
\hline Ingeniería & 59 & 398 & 195 & 11 & 788 & 58 & $\mathbf{1 . 5 0 9}$ & $\mathbf{2 3 , 8 7}$ \\
\hline Artes y humanidades & 14 & 321 & 1 & 64 & 269 & 38 & $\mathbf{7 0 7}$ & $\mathbf{1 1 , 1 8}$ \\
\hline Ciencias formales & 1 & 145 & 1 & 37 & 141 & 11 & $\mathbf{3 3 6}$ & $\mathbf{5 , 3 2}$ \\
\hline \multicolumn{1}{c}{ TOTAL } & $\mathbf{1 4 6}$ & $\mathbf{2 . 1 7 9}$ & $\mathbf{3 0 7}$ & $\mathbf{4 8 6}$ & $\mathbf{2 . 8 6 7}$ & $\mathbf{3 3 6}$ & $\mathbf{6 . 3 2 1}$ & $\mathbf{1 0 0}$ \\
\hline
\end{tabular}

* La UNED presenta un Instituto de investigación multidisciplinar (El "Instituto Universitario de Investigación") no adscrito a ningún área, por ello, el número de ítems totales de esta universidad es de 336, en lugar de 337.

Tabla VII. Distribución de URLs de instituciones por tipo de entidad

\begin{tabular}{lcccc}
\hline \multicolumn{1}{c}{ TIPO DE INSTITUCIONES } & sDOM & sDIR & N & (\%) \\
\hline Grupos de investigación & 738 & 4.678 & $\mathbf{5 . 4 1 6}$ & 40,37 \\
\hline Departamentos universitarios & 577 & 3.468 & $\mathbf{4 . 0 4 5}$ & 30,15 \\
\hline Facultades universitarias & 227 & 873 & $\mathbf{1 . 1 0 0}$ & 8,20 \\
\hline Institutos de investigación & 167 & 477 & $\mathbf{6 4 4}$ & 4,80 \\
\hline Escuelas universitarias & 213 & 364 & $\mathbf{5 7 7}$ & 4,30 \\
\hline Vicerrectorados & 35 & 415 & $\mathbf{4 5 0}$ & 3,35 \\
\hline Centros de investigación & 94 & 181 & $\mathbf{2 7 5}$ & 2,05 \\
\hline Bibliotecas universitarias & 59 & 141 & $\mathbf{2 0 0}$ & 1,49 \\
\hline Centros de estudios & 28 & 113 & $\mathbf{1 4 1}$ & 1,05 \\
\hline Asociaciones de antiguos alumnos y amigos & 18 & 108 & $\mathbf{1 2 6}$ & 0,94 \\
\hline Oficinas OTRI & 27 & 59 & $\mathbf{8 6}$ & 0,64 \\
\hline Institutos de ciencias de la educación & 24 & 51 & $\mathbf{7 5}$ & 0,56 \\
\hline Fundaciones universitarias & 35 & 36 & $\mathbf{7 1}$ & 0,53 \\
\hline Centros de formación posgrado & 15 & 54 & $\mathbf{6 9}$ & 0,51 \\
\hline Archivos universitarios & 4 & 64 & $\mathbf{6 8}$ & 0,51 \\
\hline Centros de documentación europea & 8 & 52 & $\mathbf{6 0}$ & 0,45 \\
\hline Escuelas de negocios & 6 & 8 & $\mathbf{1 4}$ & 0,10 \\
\hline & $\mathbf{2 . 2 7 5}$ & $\mathbf{1 1 . 1 4 2}$ & $\mathbf{1 3 . 4 1 7}$ & $\mathbf{1 0 0}$ \\
\hline
\end{tabular}


Tabla VIII. Ranking de universidades en función del número de URLs de instituciones

\begin{tabular}{|c|c|c|c|c|c|}
\hline $\mathbf{R}$ & UNIVERSIDAD & $\mathbf{N}$ & $\%$ & DOM & TIPO \\
\hline 1 & Universidad de Oviedo & 1.564 & 11,66 & 8 & PUB \\
\hline 2 & Universidad Internacional de Cataluña & 876 & 6,53 & 4 & PRI \\
\hline 3 & Universidad del País Vasco & 581 & 4,33 & 1 & PUB \\
\hline 4 & Universidad de Barcelona & 564 & 4,20 & 3 & PUB \\
\hline 5 & Universidad de Castilla-La Mancha & 560 & 4,17 & 5 & PUB \\
\hline 6 & Universidad Autónoma de Barcelona & 486 & 3,62 & 2 & PUB \\
\hline 7 & Universidad Carlos III de Madrid & 468 & 3,49 & 5 & PUB \\
\hline 8 & Universidad de Granada & 464 & 3,46 & 1 & PUB \\
\hline 9 & Universidad de Navarra & 414 & 3,09 & 5 & PRI \\
\hline 10 & Universidad de Santiago de Compostela & 400 & 2,98 & 1 & PUB \\
\hline 11 & Universidad Complutense de Madrid & 358 & 2,67 & 1 & PUB \\
\hline 12 & Universidad Politécnica de Cataluña & 322 & 2,40 & 3 & PUB \\
\hline 13 & Universidad de Valencia & 305 & 2,27 & 1 & PUB \\
\hline 14 & Universidad de Gerona & 302 & 2,25 & 3 & PUB \\
\hline 15 & Universidad de Extremadura & 289 & 2,15 & 1 & PUB \\
\hline 16 & Universidad de Cádiz & 259 & 1,93 & 1 & PUB \\
\hline 17 & Universidad Rovira i Virgili & 252 & 1,88 & 3 & PUB \\
\hline 18 & Universidad Oberta de Catalunya & 248 & 1,85 & 4 & PRI \\
\hline 19 & Universidad Politécnica de Valencia & 232 & 1,73 & 1 & PUB \\
\hline 20 & Universidad de Vigo & 231 & 1,72 & 1 & PUB \\
\hline 21 & Universidad de Alicante & 219 & 1,63 & 1 & PUB \\
\hline 22 & Universidad de Salamanca & 217 & 1,62 & 1 & PUB \\
\hline 23 & Universidad de Sevilla & 203 & 1,51 & 1 & PUB \\
\hline 24 & Universidad Pompeu Fabra & 189 & 1,41 & 3 & PUB \\
\hline 25 & Universidad Autónoma de Madrid & 188 & 1,40 & 1 & PUB \\
\hline 26 & Universidad de Zaragoza & 183 & 1,36 & 1 & PUB \\
\hline 27 & Universidad de Murcia & 178 & 1,33 & 1 & PUB \\
\hline 28 & Universidad Politécnica de Madrid & 174 & 1,30 & 1 & PUB \\
\hline 29 & Universidad de La Coruña & 164 & 1,22 & 1 & PUB \\
\hline 30 & Universidad de Málaga & 153 & 1,14 & 1 & PUB \\
\hline 31 & Universidad Jaume I & 146 & 1,09 & 1 & PUB \\
\hline 32 & Universidad de Valladolid & 136 & 1,01 & 1 & PUB \\
\hline 33 & Universidad de Alcalá & 131 & 0,98 & 1 & PUB \\
\hline 34 & Universidad de Las Palmas de Gran Canaria & 126 & 0,94 & 1 & PUB \\
\hline 35 & Universidad de Cantabria & 125 & 0,93 & 1 & PUB \\
\hline 36 & Universidad de Córdoba & 123 & 0,92 & 1 & PUB \\
\hline 37 & Universidad de La Laguna & 123 & 0,92 & 1 & PUB \\
\hline 38 & Universidad de las Islas Baleares & 119 & 0,89 & 2 & PUB \\
\hline 39 & Universidad de Mondragón & 119 & 0,89 & 1 & PRI \\
\hline 40 & Universidad de Lérida & 112 & 0,83 & 2 & PUB \\
\hline 41 & Universidad de Burgos & 100 & 0,75 & 1 & PUB \\
\hline 42 & Universidad de Jaén & 96 & 0,72 & 1 & PUB \\
\hline 43 & Universidad Nacional de Educación a Distancia & 82 & 0,61 & 1 & PUB \\
\hline 44 & Universidad de Almería & 75 & 0,56 & 1 & PUB \\
\hline 45 & Universidad de Huelva & 72 & 0,54 & 1 & PUB \\
\hline 46 & Universidad Politécnica de Cartagena & 69 & 0,51 & 1 & PUB \\
\hline 47 & Universidad Rey Juan Carlos & 67 & 0,50 & 2 & PUB \\
\hline 48 & Universidad Pública de Navarra & 53 & 0,40 & 1 & PUB \\
\hline 49 & Universidad de La Rioja & 46 & 0,34 & 3 & PUB \\
\hline 50 & Universidad Pontificia de Salamanca & 46 & 0,34 & 1 & PRI \\
\hline 51 & Universidad Pablo de Olavide & 44 & 0,33 & 1 & PUB \\
\hline 52 & Universidad Miguel Hernández de Elche & 43 & 0,32 & 1 & PUB \\
\hline 53 & Universidad de Deusto & 41 & 0,31 & 1 & PRI \\
\hline 54 & Universidad de León & 34 & 0,25 & 2 & PUB \\
\hline 55 & Universidad Europea Miguel de Cervantes & 30 & 0,22 & 3 & PRI \\
\hline 56 & Universidad Ramon Llull & 27 & 0,20 & 3 & PRI \\
\hline 57 & Universidad Pontificia de Comillas & 26 & 0,19 & 6 & PRI \\
\hline 58 & Universidad Católica San Antonio de Murcia & 24 & 0,18 & 1 & PRI \\
\hline 59 & Universidad de Vic & 21 & 0,16 & 2 & PRI \\
\hline 60 & Universidad Europea de Madrid & 21 & 0,16 & 1 & PRI \\
\hline 61 & Universidad Cardenal Herrera CEU & 17 & 0,13 & 3 & PRI \\
\hline 62 & Universidad San Pablo CEU & 17 & 0,13 & 3 & PRI \\
\hline 63 & IE Universidad & 11 & 0,08 & 1 & PRI \\
\hline 64 & Universidad San Jorge & 11 & 0,08 & 4 & PRI \\
\hline 65 & Universidad Antonio de Nebrija & 10 & 0,07 & 2 & PRI \\
\hline 66 & Universidad Abat Oliba CEU & 8 & 0,06 & 2 & PRI \\
\hline 67 & Universidad Alfonso X El Sabio & 8 & 0,06 & 1 & PRI \\
\hline 68 & Universidad Católica de Valencia & 6 & 0,04 & 1 & PRI \\
\hline 69 & Universidad Camilo José Cela & 2 & 0,01 & 3 & PRI \\
\hline 70 & Universidad Católica de Ávila & 2 & 0,01 & 1 & PRI \\
\hline 71 & Universidad Francisco de Vitoria & 2 & 0,01 & 1 & PRI \\
\hline 72 & Universidad Internacional de Andalucía & 2 & 0,01 & 1 & PUB \\
\hline 73 & Universidad Internacional Menéndez Pelayo & 1 & 0,01 & 3 & PRI \\
\hline 74 & Universidad a Distancia de Madrid & $\mathbf{0}$ & 0,00 & 1 & PRI \\
\hline 75 & Universidad Internacional de La Rioja & $\mathbf{0}$ & 0,00 & 1 & PRI \\
\hline 76 & Universidad Internacional de Valencia & $\mathbf{0}$ & 0,00 & 2 & PRI \\
\hline & TOTAL & 13.417 & 100 & 141 & \\
\hline
\end{tabular}


parecen no estar compensadas con el número de instituciones, lo que indica un alto uso de URLS para una misma institución. Pese a este dato, la mayoría de universidades privadas se encuentran en la cola del ranking.

\section{c) Datos comparados}

La figura 2 compara la distribución de entidades y URLs para cada una de las 76 universidades con el objetivo de visualizar las diferencias entre ambas muestras $\left(r_{s}=0,91 ; \mathrm{n}=76\right)$. La alta correlación obtenida indica que las universidades con mayor cantidad de instituciones son las que también disponen de mayor cantidad de URLs recuperados, aunque la distribución de éstos no es totalmente lineal (lo que explica su bajo coeficiente de determinación: $\left.R^{2}=0,39\right)$, debido a las prácticas de multidominio existentes, desiguales en cada espacio universitario.

La zona baja de la distribución, es decir, las universidades con pocas entidades, presentan, de forma lógica, pocos URLs. Conforme la distribución se acerca a las universidades con más ítems, el número de URLs crece de forma bastante homogé- nea. No obstante, se detectan varias universidades con una cantidad de dominios muy superior a la esperada en función de su número de entidades.

Por una parte, la UO, que pese a ser la universidad con más entidades, presenta una cantidad de URLs excesivamente alta (1.564), debido a la cantidad de direcciones web oficiales que posee (un total de 8, ya comentadas con anterioridad). Esto mismo sucede con la UCLM o la UC3M.

El sistema universitario catalán también provoca un aumento importante en número de URLs en algunas universidades como la UB, UAB y la UOC, aunque el caso más llamativo es la UIC, donde la combinación de distintos dominios e idiomas provoca que ciertas instituciones se encuentren representadas por una alta cantidad de dominios. Este hecho se comentará más detenidamente en apartados posteriores.

La figura 3 ilustra por su parte las diferencias entre entidades y URLs para cada tipo de institución, donde el coeficiente de correlación de Spearman ${ }^{11}$ entre ambas muestras es elevado $\left(r_{s}=0,98\right.$; $n=17)$.

Figura 2. Distribución del número de instituciones y URLs por universidad

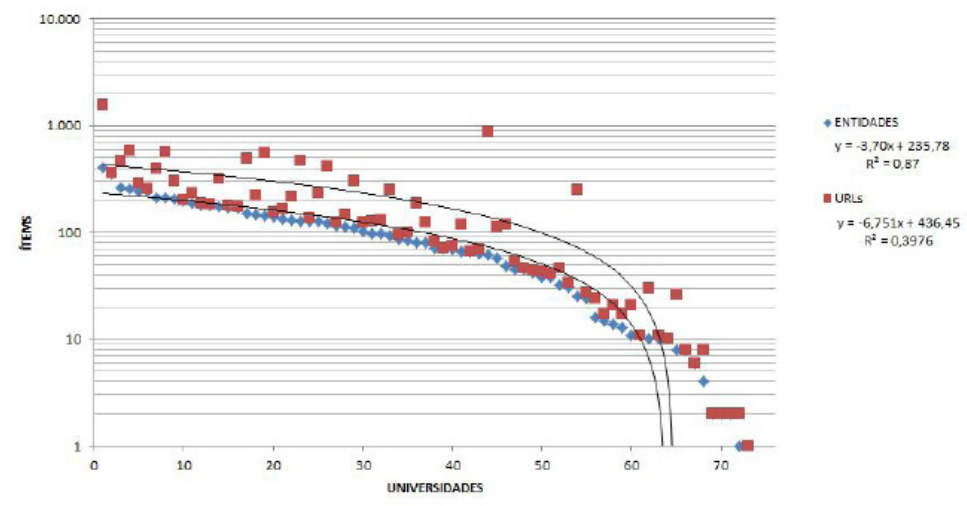

Figura 3. Número de instituciones y URLs por tipo de entidad

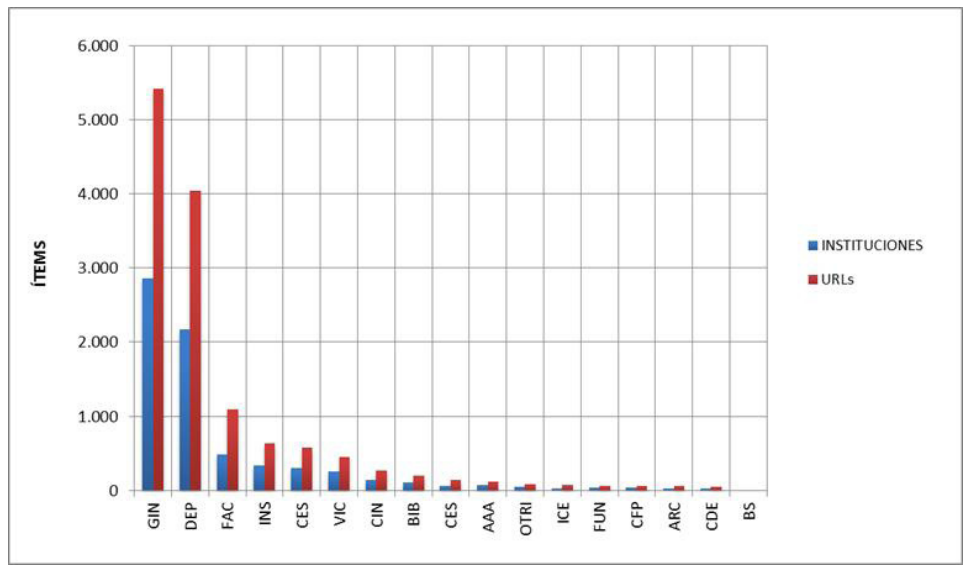




\subsubsection{Muestra de productos}

A continuación, de forma análoga a las instituciones, se procede a realizar un análisis descriptivo cuantitativo de las entidades de tipo producto.

\section{tos)}

Análisis general de entidades (produc-

Se ha identificado un total de 293 entidades ( 232 pertenecientes a universidades públicas y 60 a privadas), clasificadas en 6 tipos de productos, tal y como muestra la tabla IX.

El número total de ítems queda lejos de los 7.098 encontrados para instituciones, aunque las características de estos productos (pocos ítems por universidad), el menor número de tipos (6) y, en algunos casos (como las plataformas OCW o de vídeos), su reciente creación, justifican las diferencias.
La distribución de productos por universidad se lista en la tabla $X$, solo para aquellas universidades con más de 5 productos identificados. Solo dos universidades (UNIR y UPSA), ambas privadas, no disponen de ningún ítem. En todo caso, el número de productos por universidad es bajo, pues ninguna sobrepasa la cifra de 10.

\section{b) Análisis general de dominios web (pro-} ductos)

El espacio web correspondiente a los 293 productos genera un total de 383 URLs, cuya distribución por tipo de producto y por tipo de URL se detalla en la tabla XI. Se observa cómo los campus virtuales y los catálogos son los productos con mayor cantidad de URLs (estas entidades son también las que mayor número de ítems logran), muy por encima del resto.

Tabla IX. Distribución de productos por tipo de entidad

\begin{tabular}{lccc}
\hline \multicolumn{1}{c}{ TIPO DE PRODUCTOS } & N & \% (producto) & \% (total) \\
\hline Plataformas de campus virtual & 93 & 31,74 & 1,26 \\
\hline Catálogos y productos documentales online & 76 & 25,94 & 1,03 \\
\hline Repositorios & 37 & 12,63 & 0,50 \\
\hline Plataformas OpenCourseWare & 34 & 11,60 & 0,46 \\
\hline Plataformas de blogs & 29 & 9,90 & 0,39 \\
\hline Plataformas de vídeos & 24 & 8,19 & 0,32 \\
\hline TOTAL & $\mathbf{2 9 3}$ & $\mathbf{1 0 0}$ & $\mathbf{3 , 9 6}$ \\
\hline
\end{tabular}

Tabla X. Ranking de universidades por número de productos con sede web institucional

\begin{tabular}{clccc}
\hline $\mathbf{R}$ & \multicolumn{1}{c}{ UNIVERSIDAD } & $\mathbf{N}$ & $\mathbf{\%}$ & TIPO \\
\hline $\mathbf{1}$ & Universidad Complutense de Madrid & $\mathbf{1 0}$ & 3,42 & PUB \\
\hline $\mathbf{2}$ & Universidad de Gerona & $\mathbf{8}$ & 2,74 & PUB \\
\hline $\mathbf{3}$ & Universidad Autónoma de Barcelona & $\mathbf{7}$ & 2,40 & PUB \\
\hline $\mathbf{4}$ & Universidad de Burgos & $\mathbf{7}$ & 2,40 & PUB \\
\hline $\mathbf{5}$ & Universidad de Valencia & $\mathbf{7}$ & 2,40 & PUB \\
\hline $\mathbf{6}$ & Universidad de Zaragoza & $\mathbf{7}$ & 2,40 & PUB \\
\hline $\mathbf{7}$ & Universidad Politécnica de Valencia & $\mathbf{7}$ & 2,40 & PUB \\
\hline $\mathbf{8}$ & Universidad de Alicante & $\mathbf{6}$ & 2,05 & PUB \\
\hline $\mathbf{9}$ & Universidad de Cádiz & $\mathbf{6}$ & 2,05 & PUB \\
\hline $\mathbf{1 0}$ & Universidad de Granada & $\mathbf{6}$ & 2,05 & PUB \\
\hline $\mathbf{1 1}$ & Universidad de Huelva & $\mathbf{6}$ & 2,05 & PUB \\
\hline $\mathbf{1 2}$ & Universidad de Santiago de Compostela & $\mathbf{6}$ & 2,05 & PUB \\
\hline $\mathbf{1 3}$ & Universidad del País Vasco & $\mathbf{6}$ & 2,05 & PUB \\
\hline $\mathbf{1 4}$ & Universidad Jaume I & $\mathbf{6}$ & 2,05 & PUB \\
\hline & & & &
\end{tabular}

Tabla XI. Distribución de URLs de productos por tipo de entidad

\begin{tabular}{|c|c|c|c|c|}
\hline TIPO DE INSTITUCIONES & sDOM & SDIR & $\mathbf{N}$ & $(\%)$ \\
\hline Plataforma campus virtual & 99 & 32 & 131 & 34,20 \\
\hline Catálogos y productos documentales online & 73 & 14 & 87 & 22,72 \\
\hline Repositorios & 40 & 7 & 47 & 12,27 \\
\hline Plataforma de blogs & 22 & 20 & 42 & 10,97 \\
\hline Plataforma OpenCourseWare & 30 & 10 & 40 & 10,44 \\
\hline Plataforma de vídeos & 22 & 14 & 36 & 9,40 \\
\hline TOTAL & 286 & 97 & 383 & 100 \\
\hline
\end{tabular}


Otro dato diferente respecto al análisis de instituciones es el tipo de URL utilizada. La tabla XI indica un uso mayoritario de los subdominios (74,67\% del total de URLs de productos) frente a subdirectorios $(25,33 \%)$. Solo en las plataformas de blogs los resultados están nivelados, en el resto el uso de subdirectorios es menor.

La tabla XII ofrece por su parte el listado de universidades con más URLs correspondientes a productos.

Como se observa a partir de los datos extraídos de la tabla XII, el número de URLs de productos por universidad es bajo. De hecho, ninguna universidad alcanza los 20 URLs. Si este ranking se compara con el obtenido en la tabla VII (número de URLs de instituciones), se observan diferencias importantes, tanto en el orden de las universidades como en una mayor presencia de las universidades privadas.

\section{c) Datos comparados}

La figura 4 ejemplifica las diferencias, por tipo de producto, entre número de productos y de URLS asociados.

Tabla XII. Ranking de universidades por número de URLs de productos

\begin{tabular}{|c|c|c|c|c|}
\hline $\mathbf{R}$ & UNIVERSIDAD & $\mathbf{N}$ & $\%$ & TIPO \\
\hline 1 & Universidad de Barcelona & 16 & 4,18 & PUB \\
\hline 2 & Universidad Autónoma de Barcelona & 14 & 3,66 & PUB \\
\hline 3 & Universidad Politécnica de Cataluña & 14 & 3,66 & PUB \\
\hline 4 & Universidad Politécnica de Valencia & 11 & 2,87 & PUB \\
\hline 5 & Universidad Complutense de Madrid & 10 & 2,61 & PUB \\
\hline 6 & Universidad de Gerona & 10 & 2,61 & PUB \\
\hline 7 & Universidad de Alicante & 9 & 2,35 & PUB \\
\hline 8 & Universidad de Las Palmas de Gran Canaria & 8 & 2,09 & PUB \\
\hline 9 & Universidad Oberta de Catalunya & 8 & 2,09 & PRI \\
\hline 10 & Universidad de Burgos & 7 & 1,83 & PUB \\
\hline 11 & Universidad de Lérida & 7 & 1,83 & PUB \\
\hline 12 & Universidad de Valencia & 7 & 1,83 & PUB \\
\hline 13 & Universidad de Zaragoza & 7 & 1,83 & PUB \\
\hline 14 & Universidad del País Vasco & 7 & 1,83 & PUB \\
\hline 15 & Universidad Europea Miguel de Cervantes & 7 & 1,83 & PRI \\
\hline 16 & Universidad de Almería & 6 & 1,57 & PUB \\
\hline 17 & Universidad de Cádiz & 6 & 1,57 & PUB \\
\hline 18 & Universidad de Granada & 6 & 1,57 & PUB \\
\hline 19 & Universidad de Huelva & 6 & 1,57 & PUB \\
\hline 20 & Universidad de Navarra & 6 & 1,57 & PRI \\
\hline 21 & Universidad de Santiago de Compostela & 6 & 1,57 & PUB \\
\hline 22 & Universidad de Valladolid & 6 & 1,57 & PUB \\
\hline 23 & Universidad Jaume $I$ & 6 & 1,57 & PUB \\
\hline 24 & Universidad Politécnica de Cartagena & 6 & 1,57 & PUB \\
\hline 25 & Universidad Politécnica de Madrid & 6 & 1,57 & PUB \\
\hline 26 & Universidad Pontificia de Comillas & 6 & 1,57 & PRI \\
\hline
\end{tabular}

Figura 4. Productos y URLs para cada tipo de entidad producto

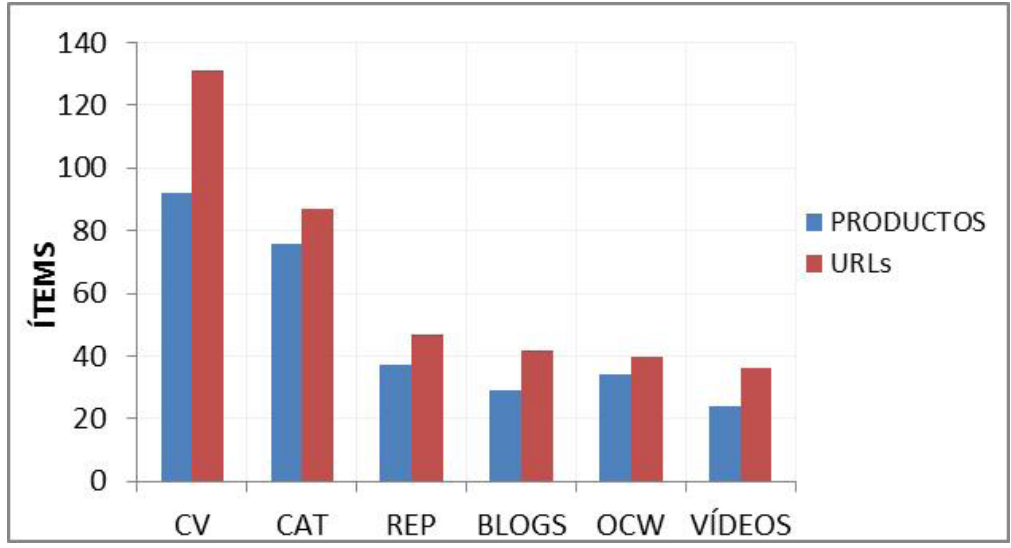


Por otra parte, la figura 5 compara, por universidad, el número de URLs correspondientes a instituciones (divididas entre 10 para adaptarlas a la escala) y productos, donde se puede observar que la correlación entre ambos muy baja $\left(r_{s}=0,55\right.$; $\mathrm{n}=76$ ), lo que refleja una política de generación de URLs diferente entre ambos tipos de entidades.

Respecto al uso de multidominios, al existir tan pocos ítems por universidad, su efecto no es tan pronunciado como en el caso de las instituciones. Las universidades cuya diferencia entre número de instituciones y número de URLs asociados es mayor son la UB (exceso de 16 URLs), UPC (exceso de 12) y UAB (exceso de 10), todas ellas catalanas.

\section{DISCUSIÓN}

El espacio académico español en línea es complejo, tanto por la cantidad de dominios alias y alternativos que poseen las universidades, como por el excesivo uso de redirecciones. El $38,16 \%$ de las universidades presenta más de 1 dominio (lo que supone un porcentaje elevado), mientras que el $19,74 \%$ presenta una redirección a otro dominio.

Este hecho provoca que las 76 universidades oficiales españolas en 2010 generen 141 URLs (más algunos otros descubiertos con posterioridad, como "muni.es" para la MU o "universitasnavarrensis. com" y "universitasnavarrensis.org", para la UNAV, entre otros), de los que $80(56,7 \%)$ son ".es".

Este relativamente bajo porcentaje puede ser debido a trabas burocráticas en la creación de dominios ".es", parcialmente flexibilizadas a raíz de la publicación de la Orden ITC/1542/2005, en la que se aprueba el Plan Nacional de nombres de dominio de Internet, y al uso de dominios alternativos.

Las prácticas de multidominio son, en proporción a su número, ligeramente más elevadas en las universidades privadas que en las públicas. El 51,72\% de las universidades con más de 1 dominio son privadas, donde se tiene que tener en cuenta además, que estas universidades suponen el 35,53\% del total del sistema universitario español.

En el caso de las redirecciones se da la misma circunstancia, el porcentaje de redireccionamiento, en proporción a su tamaño, es mayor en las universidades privadas (el $29,63 \%$ de las universidades privadas posee algún tipo de redireccionamiento, por solo el 14,29\% de las universidades públicas).

Pese a que las prácticas de multidominio y redirección son útiles como técnicas de optimización en buscadores (SEO), complican cualquier análisis de naturaleza redinformétrica, pues cada URL se considera como entidad independiente, lo que provoca dispersión en el rendimiento calculado.

Instituciones como la Universidad de Oviedo, la Universidad Carlos III de Madrid, la Universidad de Castilla-La Mancha y el sistema universitario catalán (especialmente la Universidad Internacional de Cataluña) presentan limitaciones en su análisis debido a la multiplicidad de dominios (tanto a nivel de contorno como de unidad) que provocan una dispersión en la medición de su rendimiento.

El análisis a nivel institucional de unidad revela otra característica que añade complejidad al espacio red universitario español: la naturaleza y sintaxis de los URLs. Los resultados reflejan un uso

Figura 5. Distribución comparada del número de URLs (instituciones y productos) por universidad

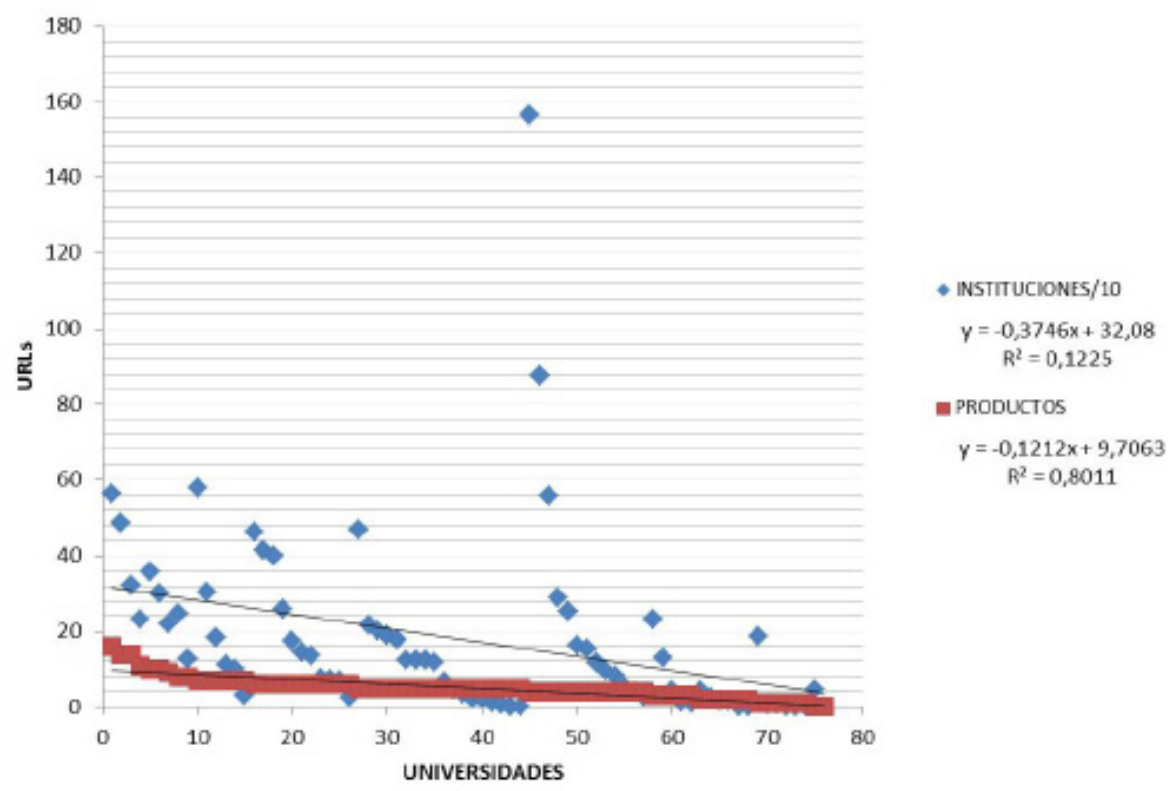


mayoritario, a nivel de unidad, de subdirectorios frente a subdominios. El $83,04 \%$ de los URLs a nivel de unidad presentan una sintaxis construida mediante subdirectorios, lo que denota cierta carencia de independencia (o madurez) de dichos espacios red dentro del sistema. Ello supone una diferencia importante respecto a las entidades de tipo producto, donde el $74,67 \%$ de URLs está construido mediante subdominios.

Esta diferencia de sintaxis de URLs entre instituciones y productos, igualmente detectada en la baja correlación obtenida en la figura $5\left(r_{s}=0,55\right)$, reflejan una clara independencia en la creación de instituciones (más centralizadas y dependientes de la política universitaria) y servicios (más descentralizados e independientes).

Por otra parte, el uso de subdirectorios genera una importante limitación metodológica en la medición de enlaces, pues Yahoo (único buscador que permite en análisis de enlaces durante el período de realización de las medidas) solo calcula enlaces al URL exacto que se le indique, y no a cada uno de los ficheros que componen dicho directorio (hecho que no ocurre en el caso de los subdominios).

Por ejemplo, la Escuela Politécnica Superior, de la UAH está representada por un URL de tipo subdirectorio ("http://www.uah.es/politecnica/"). Esto implica que Yahoo! únicamente podrá calcular enlaces exactos a este URL, y no sumará los enlaces recibidos por cada fichero englobado dentro de este subdirectorio, como sería lo lógico.

Pese a que la tasa de pérdida de enlaces no se puede conocer, existe cierta tendencia a enlazar a la página de inicio (Thelwall, 2011), por lo que las repercusiones de este hecho se minimizan en parte.

En todo caso, este hecho supone una limitación importante en el cálculo de enlaces externos a nivel interno, pues implica que las entidades analizadas reciben potencialmente más enlaces de los que Yahoo! muestra. La imposibilidad de analizar fichero a fichero los enlaces recibidos a cada sitio web es inviable por tiempo y coste, con lo que el análisis del URL general de la entidad, aunque sea subdirectorio, es la única forma de proceder.

Los grupos de investigación y los departamentos son las entidades de tipo institución que tienen un mayor número de ítems (2.867 y 2.179 respectivamente), lo que denota la importancia de estas unidades en el espacio red de la universidad (entre las dos representan el 71,01\% del total de ítems), mientras que los centros de documentación europea (28) y las escuelas de negocio (12), son las instituciones con menos ítems recopilados.

Los grupos de investigación y los departamentos son estudiados igualmente en la tesis doctoral de Ortega (2007), quien recopila un total de 2.390 URLs (sumando grupos y departamentos), mientras que en este trabajo la suma de ambas unidades -contando dominios alias y alternativos- as- ciende a 9.461 URLs. No obstante, el hecho de que Ortega no considere los dominios alternativos impide una comparación directa con sus resultados, lo que hubiera permitido estudiar el crecimiento del espacio red durante el tiempo transcurrido entre ambos trabajos.

De las entidades adscritas a campos temáticos (departamentos, grupos, escuelas, facultades, centros e institutos de investigación), se obtiene que las ciencias naturales son las más representadas en cuando a número de instituciones con URL válido para el estudio $(31,34 \%)$, seguida de las ciencias sociales $(28,29 \%)$ y de las ingenierías $(23,87 \%)$.

La preponderancia de las ciencias naturales e ingenierías ya es comentada por Ortega (2007), aunque el uso de distintas clasificaciones temáticas hace imposible de nuevo la comparación directa con su trabajo.

En cuanto a las entidades de tipo producto, destacan en número de ítems de los campus virtuales (93) y los OPACs de las bibliotecas (76), que juntos representan el $57,68 \%$ de todos los ítems de productos. No obstante, el análisis cuantitativo de ítems queda limitado por el hecho de que muchos productos solo tienen un ítem por universidad. Por ejemplo, 31 universidades poseen un solo repositorio, 34 universidades una sola plataforma OCW, 48 universidades un único OPAC y hasta 50 universidades un solo campus virtual.

Las universidades con más instituciones internas válidas para el estudio son la Universidad de Oviedo (407 ítems repartidos en 8 dominios, que representan el 5,73\% del total de instituciones), la Universidad Complutense de Madrid (348 ítems, en un solo dominio web, y que suponen el $4,90 \%$ del total de instituciones), y ya más retrasadas aparecen la Universidad de Granada (263 en 1 dominio), Universidad del País Vasco (258 ítems en 1 dominio), y la Universidad de Cádiz y Universidad de Extremadura ( 247 ítems en 1 dominio cada una).

Se observa un bajo rendimiento general de las universidades privadas, fundamentalmente en unidades de tipo institución. De las 25 universidades con menos instituciones, 23 son privadas e incluso 3 de ellas (Universidad a Distancia de Madrid, Universidad Internacional de La Rioja y la Universidad Internacional de Valencia), no consiguen ningún ítem. La primera universidad privada en cuanto a número de instituciones es la Universidad de Navarra, en el puesto 26 con 121 ítems.

El análisis de las universidades por entidades de tipo producto ofrece resultados diferentes. La universidad con más ítems es la Universidad Complutense de Madrid (10 ítems, que representan solamente el $3,42 \%$ del total), seguida por la Universidad de Gerona (con 8 ítems; 2,74\%).

Se advierte una ligera mejoría en las universidades privadas respecto al ranking de instituciones 
pues, de las 25 universidades con menor cantidad de entidades tipo producto, 7 son públicas (frente a las 2 del correspondiente ranking de instituciones), siendo la IE University la primera universidad privada en este ranking, en el puesto 15 con 5 ítems.

Los resultados obtenidos, discutidos anteriormente, son en su amplia mayoría de tipo técnico (mayor o menor uso de redirecciones y multidominios), y deben contextualizarse en relación al reflejo que suponen de las actividades y políticas universitarias. Por ejemplo, la alta presencia web de grupos y departamentos indica tanto la importancia de estas unidades dentro de la universidad, como la posibilidad de analizar los contenidos docentes y de investigación susceptibles de ser publicados en dichas sedes. Por el contrario, la ausencia web (o la presencia no adecuada) puede indicar aspectos negativos: falta de compromiso con la comunicación y publicación de contenidos, políticas universitarias determinadas y/o equivocadas, e incluso dejadez o despreocupación.

Por todo ello, y como paso previo a un análisis cuantitativo de los contenidos publicados en dichas sedes y de su impacto y consumo por parte de los usuarios (análisis de rendimiento de las sedes web), la presencia -y características- en la Red de la estructura universitaria constituye un elemento de evaluación de la calidad universitaria a tener en cuenta.

\section{CONCLUSIONES}

1. El espacio académico español en línea es excesivamente complejo, debido a las excesivas prácticas de multidominio (de 7.467 entidades universitarias de contorno y unidad identificadas, se localizan 13.941 URLs válidos) y redireccionamiento (el $19,74 \%$ de las universidades posee algún tipo de redireccionamiento en su sede en línea).

2. A nivel interno, la sintaxis de URL predominante es el subdirectorio $(83,04 \% ; 81,44 \%)$ frente al subdominio $(16,96 \% ; 18,56 \%)$, lo que provoca limitaciones metodológicas en la obtención de resultados, e indica poca madurez e independencia de dichas sedes dentro de la universidad.

3. La sintaxis de URL presenta además comportamientos diferentes en las instituciones (el 83,04\% son subdirectorios) y los productos (el $74,67 \%$ son subdominios), que indican una mayor independencia y descentralización de estos últimos.

4. Las instituciones representan el $96,04 \%$ del total de ítems a nivel de unidad universitaria. Entre éstas, los grupos de investigación (2.867 ítems, que representan el $40,39 \%$ de las entidades instituciones, y el $38,79 \%$ del total de entidades) y los departamentos (2.179 ítems, que representan el $30,70 \%$ de instituciones y el $29,48 \%$ del total de entidades) son, con diferencia, las unidades con mayor representación universitaria en la Red.
5. Entre las instituciones de tipo producto, las más representadas son los campus virtuales (93 ítems, que representan el $31,74 \%$ de productos, aunque solo el $1,26 \%$ del total de unidades) y los OPACs de bibliotecas (76 ítems, que representan el $25,94 \%$ de productos, y el $1,03 \%$ del total de entidades). Estos datos reflejan la baja proporción de productos frente a entidades, que se explica por el poco número de ítems por tipo de producto en cada universidad.

6. Las universidades privadas carecen de suficientes sedes en línea a nivel interno, fundamentalmente de entidades de tipo institución, hecho que refleja las diferencias estructurales de este tipo de instituciones frente a las universidades públicas. Esto implica que no sean correctamente representadas en la Red y que los análisis cibermétricos no aporten prácticamente información a nivel sistémico.

7. Las ciencias naturales $(31,34 \%)$ y las ciencias sociales $(28,29 \%)$ son los campos del conocimiento más representados en aquellas unidades universitarias con adscripción temática y URL válida para el estudio.

8. La Universidad de Oviedo (debido al excesivo uso de dominios alternativos) y la Universidad Internacional de Cataluña (debido a la arquitectura de la sede en línea y a la generación de URLs que no reflejan jerarquía institucional), son, durante 2010, las universidades más complejas desde un punto de vista cibermétrico.

Por último, las conclusiones obtenidas sugieren la siguiente consideración final:

El análisis descriptivo a través de las técnicas cibermétricas permite identificar y cuantificar -a nivel de unidad universitaria- las distintas instituciones y productos generados por la Universidad y, por lo tanto, permite analizar las actividades universitarias en sus distintas misiones, no solamente la puramente académica.

El estudio realizado confirma por tanto que, más allá del análisis, cuantificación e impacto de los contenidos online publicados por las universidades, existe la necesidad de analizar previamente la estructura universitaria en la Red. La mayor o menor correspondencia de la estructura institucional de la Universidad con la correspondiente estructura existente y accesible a través de Internet supone un reflejo de las distintas políticas universitarias y, por lo tanto, debería ser tenida en cuenta en su justa medida en las distintas evaluaciones de la calidad universitaria.

\section{NOTAS}

[1] http://www.ciw.cl/caracterizacion-web/estudio2007 [Fecha de consulta: 01-05-2011].

[2] http://www.educacion.gob.es/educacion/universidades/ educacion-superior-universitaria/que-estudiar-donde/ universidades-espanolas.html [Fecha de consulta: 01-05-2011]. 
[3] http://www.crue.org [Fecha de consulta: 01-05-2011].

[4] Todos los URLs consultados en: 01-05-2011.

[5] http://www.ual.es/GruposInv/FQM-230/ [Fecha de consulta: 01-05-2011].

[6] Excepto para el caso de los archivos universitarios, que por problemas técnicos solo se realizaron tomas en junio, septiembre y diciembre.

[7] http://www.idi.mineco.gob.es [Fecha de consulta: 16-092013].

[8] http://caminos.udc.es/gmni [Fecha de consulta: 01-052011]

[9] Dominios alternativos, como los de la MU (muni.es) o UNAV (universitasnavarrensis.org; universitasnavarrensis. com), no se incluyen al ser identificados con posterioridad al inicio de la investigación.

[10] Durante el período de estudio no se han localizado más de 1 URL asociada a la universidad, lo que no quiere decir que pueda existir alguna no identificada.

[11] Se emplea el coeficiente de correlación de Spearman por ser más adecuado a distribuciones no lineales que el de Pearson.

\section{BIBLIOGRAFÍA}

Adecannby, J. (2011). Web link analysis of interrelationship between top ten African universities and world universities. Annals of Library and Information Studies, vol. 58, 128-138.

Aguillo, Isidro F. (2005). Indicadores de contenidos para la web académica iberoamericana. BiD: textos universitaris de biblioteconomia i documentació, vol. 1.

Aguillo, Isidro F.; Granadino, B.; Ortega, José L.; Prieto, J.A. (2006). Scientific research activity and communication measured with cybermetrics indicators. Journal of the American Society for Information Science and Technology, vol. 57 (10), 1296-1302.

Bar-Ilan, J. (2003). The use of web search engines in information science research. Annual Review of Information Science and Technology, vol. 38, 231-288

Boudourides, M. A. ; Sigrist, B. ; Alevizos, P. D. (1999). Webometrics and the self-organization of the European information society. Rome Meeting of the SOEIS Project.

González Martín, Rubén; Aguillo, Isidro F. (1999). La presencia de las Universidades iberoamericanas en Internet: Un estudio cibermétrico en el 'cono sur'. Cuarto Taller RICYT Iberoamericano e Interamericano de Indicadores de Ciencia y Tecnología.

Heimeriks, G.; Hörlesberger, M.; Van den Besselaar, P. (2003). Mapping communication and collaboration in heterogeneous research networks. Scientometrics, vol. 58 (2), 391-413.

Kousha, K.; Horri, A. (2004). The relationship between scholarly publishing and the counts of academic inlinks to Iranian university Web sites: Exploring academic link creation motivations. Journal of Information Management and Scientometrics, vol. 1 (2), 13-22.
Li, X. (2005). National and international university departmental Web site interlinking: a webometric analysis. University of Wolverhampton: Wolverhampton (UK).

Martínez-Torres, María del Rocío; Palacios-Florencio, Beatriz; Toral-Marín, Sergio L. ; Barrero-García, Federico José. (2011). Aplicación de algoritmos genéticos a la identificación de la estructura de enlaces en portales web. Revista Española de Documentación Científica, vol. 34 (2), 232-252.

Noruzi, A. (2006). Web presence and impact factors for Middle-Eastern Countries. Online, vol. 30 (2), 22-28.

Nwagwu, Williams E.; Agarin, O. (2008). Nigerian university websites: A webometric analysis. Webology, vol. 5 (4).

Orduña-Malea, E.; Serrano-cobos, J.; Ontalba-Ruipérez, J-A. ; Lloret-Romero, N. (2010). Presencia y visibilidad web de las universidades públicas españolas. Revista Española de Documentación Científica, vol. 33 (2), 246-278.

Orduña-Malea, E.; Serrano-Cobos, J.; Lloret-Romero, N. (2009). Las universidades públicas españolas en Google Scholar: presencia y evolución de su publicación académica web. El Profesional de Información, vol. 18 (5), 493-500.

Ortega, José L. (2007). Visualización de la Web universitaria Europea: análisis cuantitativo de enlaces a través de técnicas cibermétricas. Madrid: Tesis Doctoral. Universidad Carlos III de Madrid.

Ortega, José L. ; Aguillo, Isidro F. (2007). La web académica española en el contexto del Espacio Europeo de Educación Superior: estudio exploratorio. El Profesional de la Información, vol. 16 (5), 417-425.

Ortega, J. L. ; Aguillo, Isidro F. (2008). Visualization of the Nordic academic web: Link analysis using social network tools. Information Processing \& Management, vol. 44 (4), 1624-1633.

Ortega, José L.; Aguillo, Isidro F. (2009a). Análisis estructural de la web académica iberoamericana. Revista Española de Documentación Científica, vol. 32 (3), 51-65.

Ortega, José L. ; Aguillo, Isidro F. (2009b). North America academic web space: Multicultural Canada vs. The United States homogeneity. ASIST \& ISSI PreConference Symposium on Informetrics and Scientometrics.

Ortega, José L. ; Aguillo, Isidro F. ; Cothey, V. ; Scharnhorst, A. (2008). Maps of the academic Web in the European Higher Education Area: an exploration of visual Web indicators. Scientometrics, vol. 74 (2), 295-308.

Park, H. W.; Thelwall, M. (2006). Web science communication in the age of globalization: Links among universities' websites in Asia and Europe. New Media and Society, vol. 8 (4), 631-652.

Pinto-Molina, M.; Alonso-Berrocal, J. L. ; Cordón-García, J. A. ; Fernández-Marcial, V.; García- Figuerola, C. ; García-Marco, J.; Gómez-Camarero, C.; Zazo, Á. F.; Doucet, A. V. (2004). Análisis cualitativo de la visibilidad de la investigación de las universida- 
des españolas a través de sus páginas web. Revista Española de Documentación Científica, vol. 27 (3), 345-370.

Polanco, X.; Boudourides, M.; Besagni, D.; Roche, I. (2001). Clustering and Mapping European University Web Sites Sample for Displaying Associations and Visualizing Networks. Proceeding of the NTTS\&ETK 2001 Conference. Hersonissos, Crete.

Qiu, Junping; Cheng, Jingquan; Wang, Zhi (2004). An analysis of backlinks counts and web impact factors for Chinese university websites. Scientometrics, vol. 60 (3), 463-473.

Smith, A.; Thelwall, M. (2002). Web Impact Factors for Australasian Universities. Scientometrics, vol. 54 (3), 363-380.

Tang, R.; Thelwall, M. (2003). U.S. academic departmental Web-site interlinking: disciplinary differences. Library \& Information Science Research, vol. 25 (4), 437-458.

Tang, R.; Thelwall, M. (2004). Patterns of national and international web inlinks to U.S. academic departments: an analysis of disciplinary variations. Scientometrics, vol. 60 (3), 475-485.

Thelwall, M. (2002a). A comparison of sources of links for academic web impact factor calculations. Journal of Documentation, vol. 58, 60-72.

Thelwall, M. (2002b). An initial exploration of the link relationship between UK university Web sites. ASLIB Proceedings, vol. 54 (2), 118-126.
Thelwall, M. (2002c). A research and institutional size based model for national university Web site interlinking. Journal of Documentation, vol. 58 (6), 683-694.

Thelwall, M. (2003). Web use and peer interconnectivity metrics for academic Web sites. Journal of Information Science, vol. 29 (1), 11-20.

Thelwall, M. (2011). A comparison of link and URL citation counting. ASLIB Proceedings, vol. 63 (4), 419-425

Thelwall, M.; Aguillo, I. F. (2003). La salud de las web universitarias españolas. Revista Española de Documentación Científica, vol. 26 (3), 291-305.

Thelwall, M.; Binns, R.; Harries, G. ; Page-Kennedy, T.; Price, E.; Wilkinson, D. (2002). European Union associated university Websites. Scientometrics, vol. 53 (1), 95-111.

Thelwall, M.; Tang, R.; Price, E. (2003). Linguistic patterns of academic Web use in Western Europe. Scientometrics, vol. 56 (3), 417-432.

Thelwall, M.; Vaughan, L.; Björneborn, L. (2005). Webometrics. Annual Review of Information Science and Technology, vol. 39, 81-135.

Thomas, O.; Willet, P. (2000). Webometric analysis of Departments of librarianship and information science. Journal of Information Science, vol. 26 (6), 421-428. 
ANEXO I

Sistema universitario español (2010)

\begin{tabular}{|c|c|c|c|}
\hline $\mathbf{N}$ & UNIVERSIDADES & TIPO & URL \\
\hline 1 & IE Universidad (IE) & PRI & ie.edu \\
\hline 2 & Universidad a Distancia de Madrid (UDIMA) & PRI & udima.es \\
\hline \multirow{2}{*}{3} & \multirow{2}{*}{ Universidad Abat Oliba CEU (UAO) } & \multirow{2}{*}{ PRI } & uao.es \\
\hline & & & uao.cat \\
\hline 4 & Universidad Alfonso X El Sabio (UAX) & PRI & uax.es \\
\hline \multirow{2}{*}{5} & \multirow{2}{*}{ Universidad Antonio de Nebrija (UAN) } & \multirow{2}{*}{ PRI } & nebrija.com \\
\hline & & & nebrija.es \\
\hline \multirow{2}{*}{6} & \multirow{2}{*}{ Universidad Autónoma de Barcelona (UAB) } & \multirow{2}{*}{ PUB } & uab.cat \\
\hline & & & uab.es \\
\hline 7 & Universidad Autónoma de Madrid (UAM) & PUB & uam.es \\
\hline \multirow{3}{*}{8} & \multirow{3}{*}{ Universidad Camilo José Cela (UCJC) } & \multirow{3}{*}{ PRI } & ucjc.edu \\
\hline & & & ucjc.es \\
\hline & & & universidadcamilojosecela.es \\
\hline \multirow{3}{*}{9} & \multirow{3}{*}{ Universidad Cardenal Herrera CEU (UCH) } & \multirow{3}{*}{ PRI } & uch.ceu.es \\
\hline & & & uchceu.es \\
\hline & & & ceuuch.es \\
\hline \multirow{5}{*}{10} & \multirow{5}{*}{ Universidad Carlos III de Madrid (UC3M) } & \multirow{5}{*}{ PUB } & uc3m.es \\
\hline & & & uc3m.com \\
\hline & & & uc3m.edu \\
\hline & & & uc3m.net \\
\hline & & & uc3m.org \\
\hline 11 & Universidad Católica de Ávila (UCAV) & PRI & ucavila.es \\
\hline 12 & Universidad Católica de Valencia (UCV) & PRI & ucv.es \\
\hline 13 & Universidad Católica San Antonio de Murcia (UCAM) & PRI & ucam.edu \\
\hline 14 & Universidad Complutense de Madrid (UCM) & PUB & ucm.es \\
\hline 15 & Universidad de Alcalá (UAH) & PUB & uah.es \\
\hline 16 & Universidad de Alicante (UA) & PUB & ua.es \\
\hline \multirow[t]{2}{*}{17} & Universidad de Almería (UAL) & PUB & ual.es \\
\hline & & & ub.cat \\
\hline \multirow[t]{2}{*}{18} & Universidad de Barcelona (UB) & PUB & ub.edu \\
\hline & & & ub.es \\
\hline 19 & Universidad de Burgos (UBU) & PUB & ubu.es \\
\hline 20 & Universidad de Cádiz (UCA) & PUB & uca.es \\
\hline 21 & Universidad de Cantabria (UC) & PUB & unican.es \\
\hline & & & uclm.es \\
\hline & & & uclm.com \\
\hline 22 & Universidad de Castilla-La Mancha (UCLM) & PUB & uclm.edu \\
\hline & & & uclm.net \\
\hline & & & uclm.org \\
\hline 23 & Universidad de Córdoba (UCO) & PUB & uco.es \\
\hline 24 & Universidad de Deusto (UDE) & PRI & deusto.es \\
\hline 25 & Universidad de Extremadura (UNEX) & PUB & unex.es \\
\hline & & & udg.cat \\
\hline 26 & Universidad de Gerona (UDG) & PUB & udg.edu \\
\hline & & & udg.es \\
\hline 27 & Universidad de Granada (UGR) & PUB & ugr.es \\
\hline 28 & Universidad de Huelva (UHU) & PUB & uhu.es \\
\hline 29 & Universidad de Jaén (UJA) & PUB & ujaen.es \\
\hline 30 & Universidad de La Coruña (UDC) & PUB & udc.es \\
\hline 31 & Universidad de La Laguna (ULL) & PUB & ull.es \\
\hline & & & unirioja.es \\
\hline 32 & Universidad de La Rioja (UR) & PUB & Unirioja.net \\
\hline & & & Unirioja.org \\
\hline
\end{tabular}




\begin{tabular}{|c|c|c|c|}
\hline $\mathbf{R}$ & UNIVERSIDAD & $\mathbf{N}$ & $\%$ \\
\hline \multirow{2}{*}{33} & \multirow{2}{*}{ Universidad de las Islas Baleares (UIB) } & \multirow{2}{*}{ PUB } & uib.cat \\
\hline & & & uib.es \\
\hline 34 & Universidad de Las Palmas de Gran Canaria (ULPGC) & PUB & ulpgc.es \\
\hline \multirow{2}{*}{35} & \multirow{2}{*}{ Universidad de León (UL) } & \multirow{2}{*}{ PUB } & unileon.es \\
\hline & & & unileon.org \\
\hline \multirow{2}{*}{36} & \multirow{2}{*}{ Universidad de Lérida (UDL) } & \multirow{2}{*}{ PUB } & udl.cat \\
\hline & & & udl.es \\
\hline 37 & Universidad de Málaga (UMA) & PUB & uma.es \\
\hline \multirow{2}{*}{38} & \multirow{2}{*}{ Universidad de Mondragón (MU) } & \multirow{2}{*}{ PRI } & mondragon.edu \\
\hline & & & muni.es \\
\hline 39 & Universidad de Murcia (UM) & PUB & um.es \\
\hline \multirow{7}{*}{40} & \multirow{7}{*}{ Universidad de Navarra (UNAV) } & \multirow{7}{*}{ PRI } & unav.es \\
\hline & & & unav.edu \\
\hline & & & unav.net \\
\hline & & & unav.com \\
\hline & & & unav.org \\
\hline & & & universitasnavarrensis.org \\
\hline & & & universitasnavarrensis.com \\
\hline \multirow{8}{*}{41} & \multirow{8}{*}{ Universidad de Oviedo (UO) } & \multirow{8}{*}{ PUB } & uniovi.com \\
\hline & & & uniovi.es \\
\hline & & & unioviedo.es \\
\hline & & & unioviedo.com \\
\hline & & & uniovi.org \\
\hline & & & unioviedo.org \\
\hline & & & uniovi.net \\
\hline & & & unioviedo.net \\
\hline 42 & Universidad de Salamanca (USAL) & PUB & usal.es \\
\hline 43 & Universidad de Santiago de Compostela (USC) & PUB & usc.es \\
\hline 44 & Universidad de Sevilla (US) & PUB & us.es \\
\hline 45 & Universidad de Valencia (UV) & PUB & uv.es \\
\hline 46 & Universidad de Valladolid (UVA) & PUB & uva.es \\
\hline \multirow{2}{*}{47} & \multirow{2}{*}{ Universidad de Vic (UVIC) } & PRI & uvic.cat \\
\hline & & rnt & uvic.es \\
\hline 48 & Universidad de Vigo (UVI) & PUB & uvigo.es \\
\hline 49 & Universidad de Zaragoza (UZ) & PUB & unizar.es \\
\hline 50 & Universidad del País Vasco (EHU) & PUB & ehu.es \\
\hline 51 & Universidad Europea de Madrid (UEM) & PRI & uem.es \\
\hline & & & uemc.es \\
\hline 52 & Universidad Europea Miguel de Cervantes (UEMC) & PRI & uemc.edu \\
\hline & & & uemc.org \\
\hline 53 & Universidad Francisco de Vitoria (UFV) & PRI & ufv.es \\
\hline 54 & Universidad Internacional de Andalucía (UNIA) & PUB & unia.es \\
\hline & & & uic.cat \\
\hline 55 & Universidad Internacional de Cataluña (UIC) & PRI & uic.es \\
\hline כ & - & rnt & unica.edu \\
\hline & & & unica.es \\
\hline 56 & Universidad Internacional de La Rioja (UNIR) & PRI & unir.net \\
\hline 57 & Universidad Internacional de Valencia (VIU) & PRI & viu.es \\
\hline קו & Umiversiada imernacional ae valencia (viu) & $P_{R 1}$ & fundacionviu.es \\
\hline & & & uimp.es \\
\hline 58 & Universidad Internacional Menéndez Pelayo (UIMP) & PRI & uimp.net \\
\hline & & & uimp.org \\
\hline 59 & Universidad Jaume I (UJI) & PUB & uji.es \\
\hline 60 & Universidad Miguel Hernández de Elche (UMH) & PUB & umh.es \\
\hline
\end{tabular}




\begin{tabular}{|c|c|c|c|}
\hline $\mathbf{R}$ & UNIVERSIDAD & $\mathbf{N}$ & $\%$ \\
\hline 61 & Universidad Nacional de Educación a Distancia (UNED) & PUB & uned.es \\
\hline \multirow{4}{*}{62} & \multirow{4}{*}{ Universidad Oberta de Catalunya (UOC) } & \multirow{4}{*}{ PRI } & uoc.cat \\
\hline & & & uoc.edu \\
\hline & & & uoc.es \\
\hline & & & uoc.org \\
\hline 63 & Universidad Pablo de Olavide (UPO) & PUB & upo.es \\
\hline 64 & Universidad Politécnica de Cartagena (UPCT) & PUB & upct.es \\
\hline \multirow{3}{*}{65} & \multirow{3}{*}{ Universidad Politécnica de Cataluña (UPC) } & \multirow{3}{*}{ PUB } & upc.cat \\
\hline & & & upc.edu \\
\hline & & & upc.es \\
\hline 66 & Universidad Politécnica de Madrid (UPM) & PUB & upm.es \\
\hline 67 & Universidad Politécnica de Valencia (UPV) & PUB & upv.es \\
\hline \multirow{3}{*}{68} & \multirow{3}{*}{ Universidad Pompeu Fabra (UPF) } & \multirow{3}{*}{ PUB } & upf.cat \\
\hline & & & upf.edu \\
\hline & & & upf.es \\
\hline \multirow{6}{*}{69} & \multirow{6}{*}{ Universidad Pontificia de Comillas (UPCO) } & \multirow{6}{*}{ PRI } & upcomillas.com \\
\hline & & & upcomillas.edu \\
\hline & & & upcomillas.es \\
\hline & & & upcomillas.net \\
\hline & & & upco.es \\
\hline & & & upcomillas.org \\
\hline 70 & Universidad Pontificia de Salamanca (UPSA) & PRI & upsa.es \\
\hline 71 & Universidad Pública de Navarra (UPNA) & PUB & unavarra.es \\
\hline \multirow{3}{*}{72} & \multirow{3}{*}{ Universidad Ramon Llull (URL) } & \multirow{3}{*}{ PRI } & url.cat \\
\hline & & & url.edu \\
\hline & & & url.es \\
\hline \multirow{2}{*}{73} & \multirow{2}{*}{ Universidad Rey Juan Carlos (URJC) } & \multirow{2}{*}{ PUB } & urjc.es \\
\hline & & & urjc.net \\
\hline \multirow{3}{*}{74} & \multirow{3}{*}{ Universidad Rovira i Virgili (URV) } & \multirow{3}{*}{ PUB } & urv.cat \\
\hline & & & urv.es \\
\hline & & & urv.net \\
\hline \multirow{4}{*}{75} & \multirow{4}{*}{ Universidad San Jorge (USJ) } & \multirow{4}{*}{ PRI } & usj.es \\
\hline & & & universidadsanjorge.es \\
\hline & & & universidadsanjorge.org \\
\hline & & & universidadsanjorge.net \\
\hline \multirow{3}{*}{76} & \multirow{3}{*}{ Universidad San Pablo CEU (USP) } & \multirow{3}{*}{ PRI } & uspceu.com \\
\hline & & & uspceu.es \\
\hline & & & uao.cat \\
\hline
\end{tabular}

\section{LEYENDA}

URL válida.

URL válida que redirige a otra URL válida.

URL válida pero sin acceso a recurso.

URL localizada con posterioridad al estudio 